\title{
Termination Analysis of Logic Programs through Combination of Type-Based Norms
}

\author{
MAURICE BRUYNOOGHE \\ Katholieke Universiteit Leuven, Belgium \\ and \\ MICHAEL CODISH \\ Ben-Gurion University of the Negev, Israel \\ and \\ JOHN P. GALLAGHER \\ Roskilde University, Denmark \\ and \\ SAMIR GENAIM \\ Universidad Politécnica de Madrid, Spain \\ and \\ WIM VANHOOF \\ University of Namur, Belgium
}

\begin{abstract}
This paper makes two contributions to the work on semantics based termination analysis for logic programs. The first involves a novel notion of type-based norm where for a given type a corresponding norm is defined to count in a term the number of sub-terms of that type. This provides a collection of candidate norms, one for each type defined in the program. The second enables an analyser to base termination proofs on the combination of several different norms. This is useful when different norms are better suited to justify the termination of different parts of the program. Application of the two contributions together consists in considering the combination of the typebased candidate norms for a given program. This results in a powerful and practical technique. Both contributions have been introduced into a working termination analyser. Experimentation indicates that they yield state-of-the-art results in a fully automatic analysis tool, improving with respect to methods that do not use both types and combined norms.
\end{abstract}

Categories and Subject Descriptors: D.1.6 [Logic Programming]: ; F.3.1 [Specifying and Verifying and Reasoning about Programs]: Assertions, Pre- and post-conditions

General Terms: Languages, Theory,Verification

Additional Key Words and Phrases: Abstract interpretation, dataflow analysis, global analysis, groundness analysis, program analysis, termination analysis.

\section{INTRODUCTION}

Termination analysis aims to determine that a given program definitely terminates for a specified, usually infinite, class of inputs. Proofs of termination are typi-

J. Gallagher is partly supported by the IT University, Copenhagen and by the IST programme of the European Commission, Future and Emerging Technologies under the IST-2001-38059 ASAP project. S. Genaim's work was supported by Marie Curie Fellowship number HPMF-CT-200201848 while the author was affiliated with the University of Verona. 
cally based on size functions (or level mappings) which map program states to the elements of a well founded domain. These are defined in terms of norms which measure the size of the data (or the terms). Termination is guaranteed if it can be shown that the states encountered through computation decrease in size, in particular as the program goes through its loops. In this case, if the sizes of the inputs are bounded, then so are the sizes of the initial states, which can in turn then decrease only a finite number of times in a computation. This implies that the computation for any of the specified inputs terminates.

For logic programs, loops occur through recursion and the size of a term is bounded if it is rigid. This means that the term is sufficiently instantiated so that its size does not change under further instantiation. The size of a rigid argument cannot decrease indefinitely in a computation. Analysers hence maintain two types of information: about the sizes of terms - to detect a decrease; and about their degree of instantiation - to detect rigidity.

Proving termination typically depends on finding a suitable norm and inferring sufficient size and instantiation information with respect to that norm to detect that all loops are decreasing and bounded. For practical reasons, most termination analysers choose the natural numbers as the well-founded domain and measure size using so-called linear or semi-linear norms.

The first contribution of this paper refines the way in which norms are defined. Using linear or semi-linear norms, it is difficult to treat occurrences of the same function symbol differently depending on its context in a term. Type information is considered in order to derive a (finite) collection of candidate type-based norms for the terms in a given program. The novelty is to define, for each type in the program, a norm which measures a term by counting the number of its sub-terms of that type.

The second contribution enables an analyser to base termination proofs on size functions derived from a combination of several different norms. This is useful when different norms are best suited to justify the termination of different parts of the program. Consequently, proofs of termination for different loops may be established using different norms. In addition, extra precision can be gained from dependencies between (size and instantiation) information derived from a collection of norms.

Of particular interest is the application of the two contributions together, namely, termination analysis based on the combination of those type-based norms corresponding to the types defined in a program (component). This leads to a powerful and practical technique which does not require intervention of a user in the selection of a norm. Identifying candidate norms without the intervention of a user is often considered the main missing link in automatic termination analysis for logic programs [Decorte et al. 1993]. The combination of both techniques is simple to implement, yet works well in practice. It overcomes limitations encountered with previous work when applying different norms to different parts of a program and is able to prove termination in cases where previous proposals fail.

Previous work recognises that type information provides a useful insight into the problem of guessing a norm [Bossi et al. 1992; Decorte et al. 1993; Martin et al. 1996; Decorte et al. 1996; 1997] as recursive types represent recursive data-structures and thus identify potential sources of infinite recursion. As those works aimed at finding a single norm that is suited to prove termination of all loops in the program, 
their candidate norms were more complex than ours. Using a norm as simple as counting the number of subterms of the same type as we proposed in [Vanhoof and Bruynooghe 2002] has, to the best of our knowledge, not been considered before. In fact, it is only by merging this technique with the technique of combining several norms [Genaim et al. 2002] that a powerful technique is obtained.

The idea of combining norms has been suggested before by King et al. [King et al. 1997] in the context of lower-bound time-complexity analysis. We build on that idea, applying it to termination analysis and providing a formal justification.

Both of the techniques proposed in this paper, as well as their combination, can be implemented as a preprocessing step. The result of this step is a transformed program which can then be analysed by any standard termination analyser for logic programs. Both techniques have been integrated into the TerminWeb analyser [Taboch et al. 2002; Codish and Taboch 1999]. Experimentation indicates that combining together (a subset of) the type-based candidate norms corresponding to the types defined in a program works well in practice and overcomes problems encountered in previous work. The examples presented in this paper can be accessed and run on the analyser at http://www.cs.bgu.ac.il/ mcodish/ TerminWeb/benchmarks.

Section 2 reviews the required background material on termination analysis and types for logic programs. The subsequent two sections introduce the main contributions of this paper: Section 3 introduces a specific notion of type-based norm and Section 4 describes how norms can be combined. Experimental evaluation is described in Section 5. Related work is reviewed in Section 6 and finally, Section 7 concludes. This paper is an extended version of previous work [Vanhoof and Bruynooghe 2002; Genaim et al. 2002] corresponding to the two contributions.

\section{PRELIMINARIES}

This section introduces the required background material as well as a number of motivating examples. Section 2.1 describes the basics of termination analysis and Section 2.2 introduces the required background on types. Section 2.1 opens in Section 2.1.1 with a language independent setting in which the difference between global and local termination conditions is clarified. The correctness of the latter is proven in a proposition that relies on Ramsey's theorem [Ramsey 1930]. Section 2.1.2 recalls the semantic basis for termination of logic programs and Section 2.1.3 recalls the norms used to monitor size decreases over loops. Section 2.1.4 shows the principles of termination analysis based on program abstraction and how the general setting of Section 2.1.1 is applied for logic programs. Section 2.1.5 discusses a number of examples; they illustrate the weaknesses of current approaches and provide a motivation for our work.

We assume familiarity with logic programming concepts [Lloyd 1987; Apt 1990] and consider universal termination for pure logic programs using Prolog's leftmost selection rule. We restrict attention to the case where unifications do not violate the occurs check. Terms are assumed to be constructed from a fixed set of function symbols $\Sigma$ and an infinite enumerable set of variables $V$. A term which contains no variables is said to be ground.

\subsection{Proving Termination: Background and Motivation}


2.1.1 Global versus local Termination. In the classic approach to proving termination, one should identify a mapping from program states to the elements of a well founded domain $D$ and a ranking function $f$ measuring the size of program states such that the size decreases as execution proceeds. In logic programming, this translates into the notion of acceptability [Apt and Pedreschi 1993]. Automated methods, however, do not consider all program points. They focus on the loops in the program as present in the finite graph representing its flow of control. It is sufficient to monitor that the function $f$ shows a decrease each time the control passes though some program point of each loop. The same function $f$ is used to show a decrease for all loops in the program, hence it may be viewed as a global termination condition. In logic programming, this approach was introduced in [De Schreye et al. 1992].

The analyzer upon which we base the results of this paper takes an alternative approach based on the application of local termination conditions. In this approach, under a slightly different notion of "loop", termination is guaranteed if for each loop in the program a (possibly different) ranking function is shown to decrease when execution goes through that loop. The idea of applying local termination conditions originates in the TermiLog system [Lindenstrauss and Sagiv 1997]. It is used in the TerminWeb analyser [Taboch et al. 2002] and is also considered in the functional programming setting as described in [Lee et al. 2001].

To illustrate the approach, let us assume that the program under consideration has a structural operational semantics (or small-step semantics), in which a computation is a sequence of configurations. A configuration can be viewed as a snapshot of the computation state, containing (at least) an indication to the part of the program currently being executed, and the values of the program variables (as well as possibly other components such as a stack, local naming environments and so on). For our purposes we just assume that the essential information in a configuration is a pair $\langle p, \bar{t}\rangle$ where $p$ is a program point and $\bar{t}$ represents the values of the program variables that could be changed in the transition to the next configuration. The exact form of the "program point" depends on the programming language under consideration: in an imperative language it could be the statement being executed, in assembly language it could be the value of the program counter, and in a functional language it could be the name of the function being evaluated. In all cases there is a finite number of possible program points for a given program. In our context, with the application to logic programming in mind, a program point is the name of a predicate and so we also refer to a configuration $\langle p, \bar{t}\rangle$ as a call and denote it as $p(\bar{t})$.

A computation for a program $P$ from an initial configuration $a_{0}$ can be characterised by the corresponding sequence $a_{0} \leadsto a_{1} \leadsto a_{2} \leadsto a_{3} \leadsto \cdots$ of configurations encountered in the computation. Given a notion of computation we focus on the set of possible configurations and on a binary relation which specifies how computation proceeds from configuration to configuration.

DEFinition 2.1. (calls, calls_to relation, loop) Let $P$ be a program and $S$ a (typically infinite) set of initial configurations. The set of configurations occurring in the computations of $P$ which start at an initial configuration from $S$ is denoted calls $(P, S)$. We denote by $\leftarrow\langle P, S\rangle$ the binary relation on configurations such that 
$a \leftarrow\langle P, S\rangle b$ if and only if $a \in \operatorname{calls}(P, S)$ and there is a computation starting from an initial configuration $a_{0} \in S$ of the form $a_{0} \leadsto \cdots \leadsto a \leadsto \cdots \sim b$. We refer to $\leftarrow\langle P, S\rangle$ as the calls_to relation. A pair of the form $p(\bar{s}) \leftarrow\langle P, S\rangle p(\bar{t})$ in the calls_to relation indicates a computation that revisits the same program point $p$ and is called a loop.

We will refer to the ranking function applied to measure the size of a program state as a level-mapping.

DeFinition 2.2. (level mapping, termination condition) A level mapping is a function from configurations to a well founded domain $(D,<)$. In the following we use both the order relation $<$ and its inverse $>$ when appropriate. A level mapping $f$ is a termination condition for a loop $p(\bar{s}) \leftarrow p(\bar{t})$ if $f(p(\bar{s}))>f(p(\bar{t}))$.

The following proposition establishes the basis for termination proofs using local termination conditions.

Proposition 2.1. Let $P$ be a program, $S$ a set of initial configurations and $L$ a finite set of level mappings. If for every loop $\ell$ in $\leftarrow_{\langle P, S\rangle}$, there is a level mapping from $L$ which is a termination condition for $\ell$ then every computation in $P$ starting from a configuration in $S$ is finite.

Proposition 2.1 is a general result which is applied below in Proposition 2.2 to provide the formal foundation for the TerminWeb analyser and to provide the foundation for the results established in Sections 3 and 4.

The original works on termination analysis based on local termination conditions [Lindenstrauss and Sagiv 1997; Codish and Taboch 1999] lack details in their formal justification. The first fully formal treatment for this approach is due to Dershowitz et al. [Dershowitz et al. 2001] and is based on the application of Ramsey's Theorem [Ramsey 1930]. The result of Dershowitz et al. went unnoticed and was later rediscovered independently as reported in [Lee et al. 2001; Lee 2002] and in [Codish et al. 2003]. We present here a proof in a general setting based on Ramsey's Theorem [Ramsey 1930]. For a more thorough presentation of Ramsey's Theorem see [Graham et al. 1980].

Theorem 2.1. (Ramsey's Theorem) Let $\mathcal{A}=\{\langle a, b\rangle \mid a, b \in \mathbb{N}$ and $a<b\}, \mathcal{L}$ be a finite set of colours and let $\mathcal{F}: \mathcal{A} \mapsto \mathcal{L}$ be a function associating the elements of $\mathcal{A}$ with colours from $\mathcal{L}$. Then there is a colour $f \in \mathcal{L}$ and an infinite set $\mathcal{X} \subseteq \mathbb{N}$ such that $\mathcal{F}(\langle a, b\rangle)=f$ for each $a, b \in \mathcal{X}$ for which $a<b$.

Proof (of Proposition 2.1). Assume the premise of the proposition and, in contradiction, that there is an infinite computation (sequence of configurations) $\mathcal{C}$ in $P$ starting from an initial call from $S$. Since there is a finite number of program points in $P$, there must be a program point $p$ which is visited infinitely often in $\mathcal{C}$. Denote the infinite subsequence of $\mathcal{C}$ of calls to $p$ as $\mathcal{C}_{p}=a_{1}, a_{2}, a_{3}, \ldots$. By definition, for each pair of natural numbers $i, j$ with $i<j, a_{i} \leftarrow{ }_{(P, S)} a_{j}$ is a loop and by assumption each loop is associated with a level mapping from $L$ which is a termination condition for that loop.

By Ramsey's Theorem, taking level mappings as colours, there exists some fixed level mapping $f \in L$ mapping configurations to a well founded domain $(D,<)$ and an infinite set $\mathcal{X}$ of natural numbers such that for each $i, j \in \mathcal{X}$ for which $i<j$, 
the loop $a_{i} \leftarrow_{(P, S)} a_{j}$ is assigned this level mapping $f$ which is also a termination condition.

The elements of $\mathcal{X}$ determine an infinite subsequence $\mathcal{C}_{p}^{\prime}=a_{i_{1}}, a_{i_{2}}, a_{i_{3}}, \ldots$ of $\mathcal{C}_{p}$. This gives an infinite decreasing sequence of values $f\left(a_{i_{1}}\right)>f\left(a_{i_{2}}\right)>f\left(a_{i_{3}}\right)>\cdots$ in contradiction to the well foundedness of the domain $D$.

In practical terms local termination conditions typically take a simpler form and are easier to infer automatically than global ones. In particular, there are situations where a global termination condition will involve tuples of values ordered lexicographically where each element in the tuple is a measure for an element in the state. In contrast, the local termination condition will at worst be a sum of the measures of elements from the state [Codish and Genaim 2003]. This is the case in Example 2.3 (below) where a global termination condition takes the form $f(\operatorname{ackermann}(x, y, z))=\langle x, y\rangle$ and can be seen to decrease with respect to the lexicographic ordering for both loops in the program. On the other hand, the local conditions $f_{1}(\operatorname{ackermann}(x, y, z))=y$ and $f_{2}(\operatorname{ackermann}(x, y, z))=x$ do not require us to reason about lexicographic orders and decrease respectively for the two individual loops.

While it can be shown that the existence of local termination conditions guarantees that a corresponding global condition exists, existing termination analysers based on global conditions do not always find these corresponding global conditions.

2.1.2 A Semantic Basis for Termination of Logic Programs. In the logic programming setting a configuration $p(\bar{t})$ represents a call to predicate $p$ (the program point) with arguments $\bar{t}$ (the state at that program point). The set of initial configurations corresponds to a set of initial (atomic) queries. Given a logic program $P$ and a set $S$ of initial atomic queries, the initial configurations, the computational model of logic programs under the left-to-right selection rule determines a set calls $(P, S)$ and a relation $\leftarrow_{(P, S)}$ in the terminology of the general setting of Section 2.1.1. The former is the set of all atoms $A$, modulo variable naming, such that a variant of $A$ is a selected atom under the left-to-right selection rule in some derivation for an initial query. The latter is the binary relation relating pairs of atoms encountered along the derivations of the program from an initial query.

Level mappings in the logic programming context were originally defined as mappings from ground atoms to natural numbers. An atom $A$ is bounded with respect to a level mapping $f$ if the values of $f$ are bounded on the set of $A$ 's ground instances; $A$ is rigid with respect to $f$ if all of its ground instances are mapped to the same value. In our approach level mappings apply to arbitrary atoms (configurations). To have a level mapping that is defined for all atoms, we define $f(A)$ as the minimum value of $f$ on all ground instances of $A$.

In [Apt and Pedreschi 1993], it is shown that acceptability is a sufficient and necessary criterion for termination of a call to a program executed under the leftto-right computation rule. A program $P$ is acceptable if there exists a level mapping $f$ and a model $I$ for $P$ such that for each ground instance $h \leftarrow b_{1}, \ldots, b_{n}$ of a clause in $P: f\left(b_{j}\right)<f(h)$ whenever $I \models b_{1}, \ldots, b_{j-1}$. Note that the latter means that termination of $\leftarrow b_{1}, \ldots, b_{j-1}$ and hence calling $b_{j}$ is consistent with the model. A call is terminating if and only if it is bounded under a level mapping for which the 
program is acceptable. Hence, proofs of termination involve finding an appropriate level mapping to demonstrate acceptability. However, the approach is global as the same level mapping is used for all atoms with the same predicate symbol. We adopt an alternative approach introduced in [Codish and Taboch 1999] which applies a semantics defined in terms of binary unfolding that makes termination observable. This approach supports local termination conditions.

The binary unfolding semantics of program $P$, denoted unf $(P)$, is a (typically infinite) set of binary clauses. Syntactically, a set of binary clauses of the form $p(\bar{t}) \leftarrow q(\bar{s})$ defines a binary relation on calls. However, unf $(P)$ is more general than the corresponding calls_to relation for logic programs. It provides a "goalindependent" representation both for the set $\operatorname{calls}(P, S)$ as well as for the relation $\leftarrow\langle P, S\rangle$. The semantics is goal-independent in the sense that for any set $S$ of initial queries, $\operatorname{calls}(P, S)$ and $\leftarrow\langle P, S\rangle$ are both determined from unf $(P)$ as follows:

$$
\begin{gathered}
\operatorname{calls}(P, S)=\left\{\begin{array}{l|l}
B \theta & \begin{array}{l}
A \in S, A^{\prime} \leftarrow B \in \operatorname{unf}(P), \\
\theta=\operatorname{mgu}\left(A, A^{\prime}\right)
\end{array}
\end{array}\right\} \\
\leftarrow\langle P, S\rangle=\left\{\begin{array}{l|l}
(A \theta, B \theta) & \begin{array}{l}
A \in \operatorname{calls}(P, S), A^{\prime} \leftarrow B \in \operatorname{unf}(P), \\
\theta=\operatorname{mgu}\left(A, A^{\prime}\right)
\end{array}
\end{array}\right\}
\end{gathered}
$$

We note that the binary unfolding semantics is closed under unfolding. This means that the presence of $p(\bar{r}) \leftarrow q(\bar{s})$ and $q\left(\bar{s}^{\prime}\right) \leftarrow h(\bar{t})$ implies the presence of $(p(\bar{r}) \leftarrow$ $h(\bar{t})) \theta$ with $\theta=\operatorname{mgu}\left(q(\bar{s}), q\left(\bar{s}^{\prime}\right)\right)$.

The binary unfolding semantics for logic programs was first introduced in [Gabbrielli and Giacobazzi 1994] where it was shown to make calls observable as expressed in Equation(1). It was further investigated in [Codish and Taboch 1999] where it was shown that a program $P$ has the same termination behaviour as its binary unfolding semantics unf $(P)$ and the result of Equation(2) was shown to provide a semantic basis for termination analysis. A termination analyser is obtained by approximating this semantics with respect to information about the sizes of terms and their instantiation.

2.1.3 Size and Rigidity. In the logic programming context, the state of a configuration is represented by the arguments of the call. To measure them, one usually employs the notion of a norm which maps terms to natural numbers. So-called semi-linear norms [Bossi et al. 1991] define the size of a term as the sum of the sizes of some of its arguments. More precisely, a semi-linear norm $|\cdot|$ is a mapping from terms to the natural numbers that is defined recursively: for each function symbol $f / n$ in the underlying signature there is an equation of the form

$$
\left|f\left(t_{1}, \ldots, t_{n}\right)\right|=c_{f}+\Sigma_{i \in I_{f}}\left|t_{i}\right|
$$

where constant $c_{f}$ and indices $I_{f} \subseteq\{1, \ldots, n\}$ are determined by the symbol $f / n$; and for a variable $X,|X|=0$.

Examples of well-known norms are the term-size (ts) norm, which counts the number of functors in a term, and the list-length (ll) norm, which counts the number of elements in a list. These are defined as: 


$$
\begin{gathered}
\|_{t s}= \begin{cases}1+\sum_{i=1}^{n}\left|t_{i}\right|_{t s} & \text { if } t=f\left(t_{1}, \ldots, t_{n}\right) \\
0 & \text { otherwise }\end{cases} \\
\|_{l l}= \begin{cases}1+|t s|_{l l} & \text { if } t=[t 1 \mid t s] \\
0 & \text { otherwise }\end{cases}
\end{gathered}
$$

A term is rigid for a given norm if this norm maps all of its instances to the same size. The terms $[a, b, c]$ and $\left[x_{1}, x_{2}, x_{3}\right]$ ( $a, b, c$ denote constant symbols and $x_{1}, x_{2}, x_{3}$ are variables) are rigid with respect to the list-length norm but only the first is rigid with respect to the term-size norm. One may observe that a term is rigid with respect to the term-size norm if and only if it is ground.

Termination analysers for logic programs maintain two types of information: about the sizes of terms - to detect a decrease; and about their degree of instantiation - to detect rigidity.

Instantiation (or rigidity) information is represented in the domain Pos of positive Boolean functions. The domain elements are interpreted as instantiation dependencies with respect to the given norm. For example, a formula of the form $x \wedge(y \rightarrow z)$ describes a program state in which $x$ is definitely bound to a rigid term and there exists an instantiation dependency such that whenever $y$ becomes bound to a rigid term then so does $z$. Details on Pos can be found in the literature [Marriott and Søndergaard 1993].

Size relations express linear information about the sizes of terms with respect to a given norm function. Formulas such as $x+y=z$ and $x \leq y \wedge y \leq z$ describe program states containing three terms, whose sizes $x, y$ and $z$ are related according to the given formulas. Several methods for inferring such size relations are described in the literature [Benoy and King 1996; Brodsky and Sagiv 1989; Cousot and Halbwachs 1978; De Schreye and Verschaetse 1995; Karr 1976]. They differ primarily in the techniques they apply to ensure that analyses remain finite as the abstract domain of size relations contains infinite chains.

2.1.4 Abstracting the Binary Unfolding Semantics. Termination analysis for logic programs can be based on an approximation specifying for the calls that arise in a computation, which are their rigid arguments, and for the loops in computations, which are the arguments that decrease in size. Such an approximation is obtained by abstracting the binary unfolding semantics.

The input to this process is a program $P$, a norm function $\psi_{a}$, and an initial call pattern $G^{a}$. The initial call pattern describes a set $S$ of initial calls to $P$ and is represented as a formula $p(\bar{x}) \leftarrow \psi$ where $\psi$ is a conjunction of variables from $\bar{x}$ specifying the arguments of $p$ which are required to be rigid with respect to $H_{a}$. For example, the call pattern append $\left(x_{1}, x_{2}, x_{3}\right) \leftarrow x_{1} \wedge x_{2}$, which can be abbreviated as append(true, true, $\left.x_{3}\right)$, describes the set of calls to append in which (at least) the first two arguments are rigid with respect to the chosen norm. We say that a call pattern $G^{a}$ is rigid with respect to a level mapping $f$ if all calls described by 
$G^{a}$ are rigid with respect to $f$. Basically this means that $f$ is defined in terms of the sizes of rigid arguments in the call pattern.

The outcome of the abstraction process is: (1) a finite set of call patterns, denoted $\operatorname{calls}_{a}\left(P, G^{a}\right)$, which approximates the set calls $(P, S)$. Each element in this set is of the form $p(\bar{x}) \leftarrow \psi$ with the same interpretation as for the initial call pattern $G^{a}$; and (2) a finite set of abstract binary clauses, denoted $u n f_{a}(P)$ which approximates the set unf $(P)$. Each abstract binary clause is of the form $p(\bar{x}) \leftarrow \mu(\bar{x}, \bar{y}), q(\bar{y})$ where $\bar{x}$ and $\bar{y}$ are tuples of distinct variables and $\mu(\bar{x}, \bar{y})$ is a conjunction of constraints on the variables of $\bar{x}$ and $\bar{y}$. The abstract binary clause $p(\bar{x}) \leftarrow \mu(\bar{x}, \bar{y}), q(\bar{y})$ describes a concrete binary clause $p(\bar{s}) \leftarrow q(\bar{t})$ with respect to the norm $\|_{a}$ if the instance $\mu\left(|\vec{s}|_{a},||_{a}\right)$ of constraint $\mu(\bar{x}, \bar{y})$ holds. There is a concrete binary clause $p(\bar{s}) \leftarrow q(\bar{t})$ iff $p(\bar{s})=p(\bar{x}) \theta$ where $\bar{x}$ is a tuple of distinct variables and there is an SLD derivation of non-zero length from $\leftarrow p(\bar{x})$ to $\leftarrow q(\bar{t}), Q$ with computed answer $\theta$. The set of concrete binary clauses described by an abstract one is closed under instantiation. Namely, if $\mu\left(|\vec{s}|_{a},||_{a}\right)$ holds then so does $\mu\left(|\bar{s} \theta|_{a},|\bar{t} \theta|_{a}\right)$ for any substitution $\theta$.

Different analyzers assume different restrictions on the constraints in $\mu(\bar{x}, \bar{y})$. For simplicity, we will assume that $\mu(\bar{x}, \bar{y})$ is a conjunction of binary relations $(<, \leq,=)$ between the variables of $\bar{x}$ and $\bar{y}$ and that all variables are constrained to be nonnegative, although in general (and in the TerminWeb analyser) no such restriction is necessary. This is the choice made in the TermiLog analyser of Lindenstrauss and Sagiv [Lindenstrauss and Sagiv 1997], where such constraints are termed monotonicity constraints. A similar choice is made in the analyser of Lee, Jones and Ben-Amram [Lee et al. 2001], where such constraints are represented as size change graphs. An example of an abstract binary clause (taken from Example 2.1) is as follows:

$$
\operatorname{append}(\mathrm{x}, \mathrm{y}, \mathrm{z}) \leftarrow[\mathrm{u}<\mathrm{x}, \mathrm{y}=\mathrm{v}, \mathrm{w}<\mathrm{z}], \operatorname{append}(\mathrm{u}, \mathrm{v}, \mathrm{w}) .
$$

We do not detail here the technique applied to compute these approximations. The interested reader can refer to [Codish and Taboch 1999]. For intuition we describe briefly the approach which is based on a technique termed abstract compilation (as for example in [Codish and Taboch 1999; Lindenstrauss and Sagiv 1997; Mesnard and Neumerkel 2001]). The program $P$ to be analysed is first abstracted, using the chosen norm, to a corresponding abstract program $P^{a}$ which is obtained by systematically replacing predicate arguments in $P$ by corresponding abstract arguments. An argument $t$ is replaced by its term abstraction $\left.\right|_{a} ^{\alpha}$ which is the same as $\|_{a}$ except that its value on a variable $x$ is a size variable $x^{\prime}$, instead of zero. For example, $\mid\left[x_{1} \mid x_{2}\right]_{l l}^{\alpha}=1+x_{2}^{\prime}$, and $\mid\left[x_{1} \mid x_{2}\right]_{t s}^{\alpha}=1+x_{1}^{\prime}+x_{2}^{\prime}$. The primed variables range over term sizes, with respect to the considered norm.

An atom with all its arguments abstracted is called an abstract atom. As variable names are of no concern, often we "reuse" the variables of an atom as size variables, instead of introducing primed variables. For example, the abstract atom corresponding to $p([x \mid y],[x \mid z])$ with respect to the list-length norm is usually written $p(1+y, 1+z)$.

The set of abstract binary clauses $u n f_{a}(P)$ can be obtained as the binary unfolding semantics of an abstract program $P^{a}$. The program $P^{a}$ is an abstraction of the concrete program $P$, in the sense that whenever $p\left(t_{1}, \ldots, t_{n}\right)$ is a ground 
consequence of $P$, then $p\left(\left|t_{1}\right|, \ldots,\left|t_{n}\right|\right)$ is a consequence of $P^{a}$. The intuition of size dependencies is that where program variables range over terms, size variables range over the sizes of terms and where a program predicate $p / n$ represents an $n$-ary relation on terms, the corresponding abstract predicate represents an $n$-ary relation on the sizes of terms. For a tuple $\bar{t}=t_{1}, \ldots, t_{n}$ and a norm $|\cdot|$ we write $t_{t}$ to denote the tuple $\left|t_{1}\right|, \ldots,\left|t_{n}\right|$.

The following proposition, a rephrasing of the result introduced in [Codish and Taboch 1999], establishes conditions on any set $u n f_{a}(P)$ of abstract binary clauses that approximates unf $(P)$, which imply termination in unf $(P)$ and hence in $P$ itself.

Proposition 2.2. Let $P$ be a logic program, $\|_{a}$ a norm, and $G^{a}$ an initial call pattern specifying the arguments in the initial query which are rigid with respect to $\vartheta_{a}$. Let unf $f_{a}(P)$ and calls $\left(P, G^{a}\right)$ denote approximations of unf $(P)$ and calls $(P, S)$ where $S$ is the set of initial queries described by $G^{a}$ (with respect to the given norm). If for each abstract call $p(\bar{x}) \leftarrow \psi$ in calls ${ }_{a}\left(P, G^{a}\right)$ and matching recursive abstract binary clause $p(\bar{x}) \leftarrow \mu(\bar{x}, \bar{y}), p(\bar{y})$ in unf $f_{a}(P)$ there exists a level mapping $f$ such that

(i) $p(\bar{x}) \leftarrow \psi$ is rigid with respect to $f$ and

(ii) for any tuples of concrete arguments $\bar{t}$ and $\bar{s}$ such that $\mu\left(\left|t_{a},\right| s_{a}\right)$ we have also $f(p(\bar{t}))>f(p(\bar{s}))$,

then $P$ terminates for all initial queries described by $G^{a}$.

Proof. Let $p(\bar{t}) \leftarrow\langle$ unf $(P), S\rangle p(\bar{s})$ be any loop in a computation in unf $(P)$ starting from a query described by $G^{a}$. We show that the assumptions on unf $f_{a}(P)$ imply that the loop has a terminating condition, and it then follows from Proposition 2.1 that unf $(P)$ terminates for all initial queries described by $G^{a}$. This then implies termination of all such queries in $P$, since every loop in $P$ is also a loop in unf $(P)$.

Consider the loop $p(\bar{t}) \leftarrow_{\langle\text {unf }(P), S\rangle} p(\bar{s})$. It follows that $p(\bar{t})$ is a call in calls $(P, S)$ and that there exists a renaming $p\left(\bar{t}^{\prime}\right) \leftarrow p\left(\bar{s}^{\prime}\right)$ of a binary clause in unf $(P)$ such that $p(\bar{s})=p\left(\bar{s}^{\prime}\right) \theta$ where $\theta=m g u\left(\bar{t}, \bar{t}^{\prime}\right)$. The call $p(\bar{t})$ is described by some call pattern $p(\bar{x}) \leftarrow \psi$ in calls $s_{a}\left(P, G^{a}\right)$ and the binary clause $p\left(\bar{t}^{\prime}\right) \leftarrow p\left(\bar{s}^{\prime}\right)$ is described by an abstract binary clause $p(\bar{x}) \leftarrow \mu(\bar{x}, \bar{y}), p(\bar{y})$ such that $\mu\left(\left|\bar{t}^{\prime}\right|_{a},\left|\bar{s}^{\prime}\right|_{a}\right)$. This holds for all instances, in particular, $\mu\left(\left|\bar{t}^{\prime} \theta\right|_{a},\left|\bar{s}^{\prime} \theta\right|_{a}\right)$ holds. However,

$$
\mu\left(\left|\bar{t}^{\prime} \theta\right|_{a},\left|\bar{s}^{\prime} \theta\right|_{a}\right)=\mu\left(|\bar{t} \theta|_{a},|\vec{s}|_{a}\right)
$$

By assumption (i) there is a level mapping $f$ such that $p(\bar{t})$ is rigid with respect to f. By assumption (ii)

$$
\mu\left(\left|\mathbb{t}_{a},\right| s_{a}\right) \rightarrow f(p(\bar{t}))>f(p(\bar{s}))
$$

Putting (4) and (5) together we have $f(p(\bar{t} \theta))>f(p(\bar{s}))$. But $f(p(\bar{t}))=f(p(\bar{t} \theta))$, since $p(\bar{t})$ is rigid with respect to $f$ and hence $f(p(\bar{t}))>f(p(\bar{s}))$. This demonstrates that the loop has a terminating condition, which establishes the result.

We note that there exist efficient algorithms to determine conditions under which (i) and (ii) in Proposition 2.2 hold [Codish and Taboch 1999]. Existing analyzers typically infer these automatically when they are of the form $f\left(p\left(x_{1}, \ldots, x_{n}\right)\right)=$ 
$\Sigma_{I}\left|x_{i}\right|$ for a set $I \subseteq\{1, \ldots, n\}$ of argument positions which are rigid in the given call pattern with respect to the chosen norm.

2.1.5 Examples of termination analysis. We proceed to demonstrate the application of termination analysis through a series of examples.

Example 2.1. Consider the append/3 predicate and its abstractions using the term-size and list-length norms. The concrete term [] is abstracted respectively to 1 and to 0 because $\mid\left[\|_{t s}=1\right.$ and $\mid\left[\|_{l l}=0\right.$. The concrete term $[\mathrm{X} \mid \mathrm{X} \mathbf{s}]$ is abstracted respectively to $1+\mathbf{X}_{t s}+\mathbf{X s}_{t s}$ and $1+\mathbf{X s}_{l l}$ where size variable $\mathbf{X}_{t s}$ represents the term-size of the term bound to the program variable $\mathrm{X}$ and size variables $\mathrm{X}_{\mathbf{s}_{t s}}$ and $\mathrm{X}_{l l}$ represent the term-size and list-length of the term bound to program variable Xs.

\begin{tabular}{|c|c|}
\hline term-size abstraction & list-length abstraction \\
\hline $\begin{array}{l}\text { append }\left(1, \mathrm{Ys}_{t s}, \mathrm{Ys}_{t s}\right) \\
\text { append }\left(1+\mathrm{X}_{t s}+\mathrm{X} \mathbf{s}_{t s}, \mathrm{Y} \mathbf{s}_{t s}, 1+\mathrm{X}_{t s}+\mathrm{Z} \mathbf{s}_{t s}\right):- \\
\quad \text { append }\left(\mathrm{X}_{t s}, \mathrm{Ys}_{t s}, Z \mathbf{s}_{t s}\right)\end{array}$ & $\begin{array}{l}\text { append }\left(0, Y \mathbf{s}_{l l}, Y \mathbf{s}_{l l}\right) \\
\text { append }\left(1+\mathrm{X} \mathbf{s}_{l l}, \mathrm{Y} \mathbf{s}_{l l}, 1+Z \mathbf{s}_{l l}\right):- \\
\quad \text { append }\left(X \mathbf{s}_{l l}, Y \mathbf{s}_{l l}, Z \mathbf{s}_{l l}\right) .\end{array}$ \\
\hline
\end{tabular}

The concrete append program specifies the relation $\{(x, y, z) \mid z=x . y\}$ (z equals the concatenation of $x$ and $y)$. The abstract programs specify the relations: (1) $\{(x, y, z) \mid z+1=x+y\}$ for the term-size abstraction which means that the termsize of $z$ plus one is equal to the sum of the term-sizes of $x$ and $y$, and (2) $\{(x, y, z) \mid z=x+y\}$ for the list-length abstraction which means that the listlength of $z$ is equal to the sum of the list-length of $x$ and $y$. The instantiation analyses obtained by applying a Pos analysis to the abstract programs specify the relations: (1) $\{(x, y, z) \mid(x \wedge y) \leftrightarrow z\}$ meaning that $x$ and $y$ are rigid with respect to term-size (ground) if and only if $z$ is, and (2) $\{(x, y, z) \mid x \wedge(y \leftrightarrow z)\}$ meaning that $x$ is rigid with respect to list-length (a closed list) and $y$ is so if and only if $z$ is. A termination analysis based on either of these two norms infers the single recursive binary clause in the abstract domain of size relations:

$$
\operatorname{append}(\mathrm{x}, \mathrm{y}, \mathrm{z}) \leftarrow[\mathrm{u}<\mathrm{x}, \mathrm{y}=\mathrm{v}, \mathrm{w}<\mathrm{z}], \operatorname{append}(\mathrm{u}, \mathrm{v}, \mathrm{w}) .
$$

With respect to both norms this indicates that from one recursive call to a future one, the first and third arguments decrease in size and the second remains equal in size.

For the initial call pattern $G^{a}=$ append(true,true, ${ }_{-}$) (with respect to either of the norms), instantiation analysis infers that all calls to append/3 are of the same form. Taking the level mapping $f($ append $(x, y, z))=|x|$ hence satisfies conditions (i) and (ii) of Proposition 2.2. Similarly, for the initial call pattern $G^{a}=$ append(,,, true) (with respect to either of the norms), all call patterns are of the same form and the level mapping $f(\operatorname{append}(x, y, z))=|z|$ provides the proof of termination. The analyzer identifies the existence of these level mappings automatically.

The previous example might leave the reader with the impression that finding a norm with which to prove termination is easy. Analyses based on two different 
norms were demonstrated and both lead to a proof of termination. The next example indicates that this is not always the case. Here, analysis based on term-size does not find a proof of termination, whereas analysis based on list-length does, due to a recursive call with a list whose list-length has decreased but where the term-size of an element remains the same. One should keep in mind that there are any number of norms that might be considered. A termination analyser may be programmed to consider some finite collection of alternative norms but how should these be chosen? In Section 3 we come back to this question and propose to consider one norm for each "type" in the program.

EXAMPLE 2.2. Consider the following program which together with the append/3 predicate from Example 2.1 constructs (bottom-up) a parse tree for words in the language $a^{n} b^{n}$ based on the context free grammar $s \rightarrow a s b \mid a b$. Here " $a$ " and " $b$ " are the terminals, " $s$ " is a non-terminal and a word is represented as a list of terminals. A sentential form is a list of terminals and non-terminals. Non-terminals are indicated as terms representing partial derivation trees. So, for instance, the derivation for aabb proceeds as follows: $[\mathrm{a}, \mathrm{a}, \mathrm{b}, \mathrm{b}] \sim[\mathrm{a}, \mathrm{s}(\mathrm{a}, \mathrm{b}), \mathrm{b}] \sim[\mathrm{s}(\mathrm{a}, \mathrm{s}(\mathrm{a}, \mathrm{b}), \mathrm{b})]$ terminating with a sentential form consisting of a single non-terminal, the start symbol, which represents the desired parse tree.

\begin{tabular}{|c|c|}
\hline concrete program & list-length abstraction \\
\hline $\begin{array}{l}\operatorname{parse}(\mathrm{Xs}, \mathrm{T}):- \\
\quad \operatorname{append}(\mathrm{As},[\mathrm{a}, \mathrm{s}(\mathrm{A}, \mathrm{B}, \mathrm{C}), \mathrm{b} \mid \mathrm{Bs}], \mathrm{Xs}), \\
\operatorname{append}(\mathrm{As},[\mathrm{s}(\mathrm{a}, \mathrm{s}(\mathrm{A}, \mathrm{B}, \mathrm{C}), \mathrm{b}) \mid \mathrm{Bs}], \mathrm{Ys}), \\
\operatorname{parse}(\mathrm{Ys}, \mathrm{T})\end{array}$ & 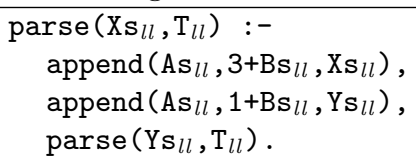 \\
\hline $\begin{array}{l}\operatorname{parse}(\mathrm{Xs}, \mathrm{T}):- \\
\quad \operatorname{append}(\mathrm{As},[\mathrm{a}, \mathrm{s}(\mathrm{A}, \mathrm{B}), \mathrm{b} \mid \mathrm{Bs}], \mathrm{Xs}) \\
\quad \operatorname{append}(\mathrm{As},[\mathrm{s}(\mathrm{a}, \mathrm{s}(\mathrm{A}, \mathrm{B}), \mathrm{b}) \mid \mathrm{Bs}], \mathrm{Ys}) \\
\operatorname{parse}(\mathrm{Ys}, \mathrm{T})\end{array}$ & $\begin{array}{l}\operatorname{parse}\left(\mathrm{X} \mathbf{s}_{l l}, \mathrm{~T}_{l l}\right):- \\
\quad \operatorname{append}\left(\mathrm{A} \mathbf{s}_{l l}, 3+\mathrm{B} \mathbf{s}_{l l}, \mathrm{X} \mathbf{s}_{l l}\right), \\
\quad \text { append }\left(\mathrm{A} \mathbf{s}_{l l}, 1+B \mathbf{s}_{l l}, \mathrm{Y} \mathbf{s}_{l l}\right), \\
\quad \operatorname{parse}\left(\mathrm{Ys}_{l l}, \mathrm{~T}_{l l}\right) .\end{array}$ \\
\hline $\begin{array}{l}\operatorname{parse}(\mathrm{Xs}, \mathrm{T}):- \\
\quad \operatorname{append}(\mathrm{As},[\mathrm{a}, \mathrm{b} \mid \mathrm{Bs}], \mathrm{Xs}) \\
\quad \operatorname{append}(\mathrm{As},[\mathrm{s}(\mathrm{a}, \mathrm{b}) \mid \mathrm{Bs}], \mathrm{Ys}) \\
\quad \operatorname{parse}(\mathrm{Ys}, \mathrm{T}) \\
\operatorname{parse}([\mathrm{s}(\mathrm{A}, \mathrm{B})], \mathrm{s}(\mathrm{A}, \mathrm{B})) \\
\operatorname{parse}([\mathrm{s}(\mathrm{A}, \mathrm{B}, \mathrm{C})], \mathrm{s}(\mathrm{A}, \mathrm{B}, \mathrm{C}))\end{array}$ & 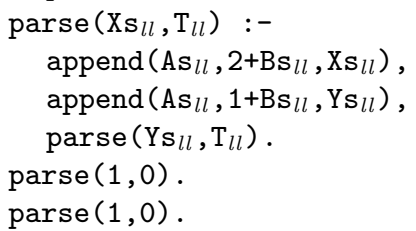 \\
\hline
\end{tabular}

Note that the second and fifth abstract clauses are identical to respectively the first and fourth abstract clauses and can be removed. A termination analysis based on the list-length norm infers that loops for parse/2 are approximated by the binary clause:

$$
\operatorname{parse}(\mathrm{x}, \mathrm{y}) \leftarrow[\mathrm{x}>\mathrm{u}, \mathrm{y}=\mathrm{v}], \operatorname{parse}(\mathrm{u}, \mathrm{v})
$$

This together with the information regarding the loops for append/3 and the instantiation information derived provides a proof of termination for all inputs of the form parse(Word, Tree) for which Word is rigid with respect to list-length (namely a closed list). Note that using the term-size norm we fail to prove termination. This is because in the third concrete clause $\left\|\mathrm{a}, \mathrm{b}\left|\mathrm{Bs} \|_{t s}=\right|\left[\mathrm{s}(\mathrm{a}, \mathrm{b}) \mid \mathrm{Bs} \|_{t s}\right.\right.$, and so with respect to this norm the corresponding abstract binary clause representing the reduction from the head to the recursive call to parse/2 is of the form $\operatorname{parse}(\mathrm{x}, \mathrm{y}) \leftarrow[\mathrm{x}=\mathrm{u}, \mathrm{y}=\mathrm{v}], \operatorname{parse}(\mathrm{u}, \mathrm{v})$. 
The next example demonstrates the advantage of proving termination by considering each of the individual loops in the program separately. With the exception of the analyser described in [Lindenstrauss and Sagiv 1997], after which our termination condition is modelled, and the analyser described in [Lee et al. 2001] which employs a similar technique, other analysers require a "global" level mapping based on lexicographic ordering to show termination for this example.

EXAMPLE 2.3. Consider the following program, on the left, which computes the Ackermann function. The analysis using the term-size norm identifies two types of loops, indicated below on the right. The proof of termination involves two simple level mappings for the ackermann/3 predicate: one which measures the size of the first argument and the other the size of the second argument.

\begin{tabular}{c|c} 
concrete program & loops wrt term-size norm \\
\hline ackermann $(0, \mathrm{~N}, \mathrm{~s}(\mathrm{~N}))$. & \\
ackermann $(\mathrm{s}(\mathrm{M}), \mathrm{O}, \operatorname{Res}):-$ & $\operatorname{ackermann}(\mathrm{x}, \mathrm{y}, \mathrm{z}):-$ \\
$\operatorname{ackermann}(\mathrm{M}, \mathrm{s}(\mathrm{O}), \operatorname{Res})$. & {$[\mathrm{x}=\mathrm{u}, \mathrm{y}>\mathrm{v}], \operatorname{ackermann}(\mathrm{u}, \mathrm{v}, \mathrm{w})$.} \\
$\operatorname{ackermann}(\mathrm{s}(\mathrm{M}), \mathrm{s}(\mathrm{N}), \operatorname{Res}):-$ & $\operatorname{ackermann}(\mathrm{x}, \mathrm{y}, \mathrm{z}):-$ \\
$\operatorname{ackermann}(\mathrm{s}(\mathrm{M}), \mathrm{N}, \operatorname{Res} 1)$, & {$[\mathrm{x}>\mathrm{u}], \operatorname{ackermann}(\mathrm{u}, \mathrm{v}, \mathrm{w})$.} \\
$\operatorname{ackermann}(\mathrm{M}, \operatorname{Res} 1, \operatorname{Res})$. &
\end{tabular}

Both loop descriptions together provide a proof of termination for all inputs that are rigid in the first two arguments.

In the more classic approach to proving termination, a global termination condition is required. For this example, the function $f(\operatorname{ackermann}(x, y))=\langle x, y\rangle$ can be seen to decrease with respect to the lexicographic ordering for both loop descriptions. This provides a proof of termination. However, current termination analysers for logic programs do not implement solvers to consider lexicographic orders. Hence the practical advantage of considering local termination conditions is that the orderings are of a simpler form.

Our fourth and last preliminary example illustrates a program for which existing technology does not give a sufficient result. We will come back to this example as we introduce our results in the coming sections.

EXAMPLE 2.4. Consider the following program which transposes the elements of a matrix. Some of the program points have been annotated (e.g., (a) to facilitate the demonstration. A matrix is represented as a list of rows where each row is a list of elements and a row in the transposed matrix corresponds to a column in the original. The relation transpose(M1,M2) holds if $\mathrm{M} 2$ is the list of columns of elements in the rows of M1. For example, transpose $([\mathrm{a}, \mathrm{b}, \mathrm{c}],[\mathrm{d}, \mathrm{e}, \mathrm{f}]]$, $[[\mathrm{a}, \mathrm{d}],[\mathrm{b}, \mathrm{e}],[\mathrm{c}, \mathrm{f}]])$ is in the relation.

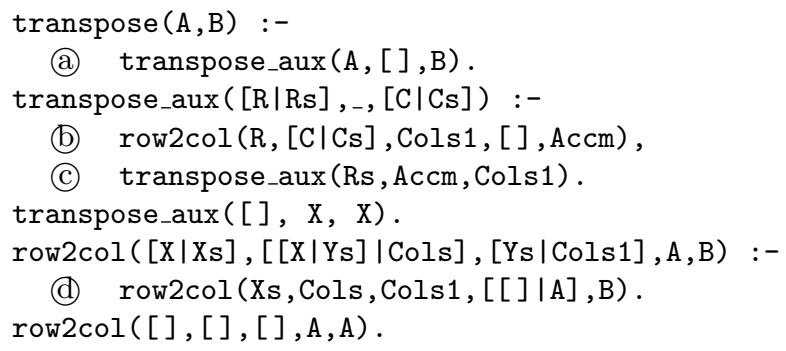


The program works as follows: If there are no rows, transpose_aux/3 returns the accumulator (its second argument) as the transposed matrix (which is [] in the initial call). Otherwise it uses row $2 \mathrm{col} / 5$ to distribute the elements of the first row over Cols1, the result of calling transpose_aux/3 with the remainder of the rows. Note that row2col/5 not only computes the transposed matrix (in its second argument), but also, in its last argument, the accumulator needed by the base case of transpose_aux/3 (a matrix with as many empty rows as there are elements in a row of the initial matrix). For the latter, it uses its own accumulator (fourth argument).

The program contains two loops: the one, for transpose_aux $/ 3$, invoked at program point (a) with the recursive calls at program point (c), and the other, for row2col/5 invoked at program point (b) with the recursive calls at program point (d).

First Observation:. The program terminates when the first argument is a ground matrix:

?- transpose([ $[a, b, c],[d, e, f]]$, Cols $)$.

Cols $=[[a, d],[b, e],[c, f]]$

Analysis applying the term-size norm delivers a proof of termination for queries in which the first argument is ground. This is because the analysis detects that the first argument in both loops is both decreasing and rigid. In the one loop, at point (a) the first argument is rigid by assumption and the decrease from $[\mathrm{R} \mid \mathrm{Rs}]$ in the head of the recursive clause to $\mathrm{R}$ in the call at point (c) is straightforward to detect. In the other loop, invoked at point (b), the first argument $\mathrm{R}$ is rigid because $[\mathrm{R} \mid \mathrm{Rs}]$ in the head is rigid and the decrease in size from $[\mathrm{X} \mid \mathrm{Xs}]$ in the head of the recursive clause to $\mathrm{Xs}$ in the call at point (d) is also straightforward to detect. The size information with respect to the term-size norm detected by the analyser is as follows:

transpose_aux $(\mathrm{A}, \mathrm{B}, \mathrm{C}) \leftarrow[\mathrm{D}<\mathrm{A}]$, transpose_aux $(\mathrm{D}, \mathrm{E}, \mathrm{F})$

$\operatorname{row} 2 \operatorname{col}(\mathrm{A}, \mathrm{B}, \mathrm{C}, \mathrm{D}, \mathrm{E}) \leftarrow[\mathrm{F}<\mathrm{A}, \mathrm{G}<\mathrm{B}, \mathrm{H}<\mathrm{C}, \mathrm{D}<\mathrm{I}, \mathrm{E}=\mathrm{J}], \operatorname{row} 2 \operatorname{col}(\mathrm{F}, \mathrm{G}, \mathrm{H}, \mathrm{I}, \mathrm{J})$

Second Observation: The program terminates when the second argument is a ground matrix:

$$
\begin{aligned}
& \text { ?- transpose( Rows, }[[a, d],[b, e],[c, f]]) . \\
& \text { Rows }=[[a, b, c],[d, e, f]]
\end{aligned}
$$

However for this case, analysis using the term-size norm does not deliver a proof of termination for queries in which the second argument is ground. The problem stems from the clause

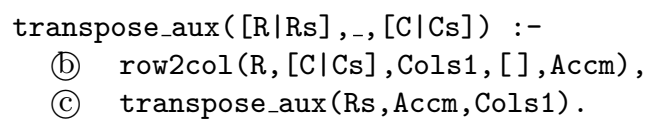

To prove termination, the analyser should detect that the term-size of $[\mathrm{C} \mid \mathrm{Cs}]$ in the head of this clause is strictly larger than that of Cols1 in the recursive call at program point (c). To do this, the analyser must be able to detect that upon success of the call to row $2 \mathrm{col} / 5$ at program point (b), the term-size of the third argument (of this call) is always strictly smaller than that of the second argument (of this call). 
To this end the analyser must reason about the success patterns of the row2col/5 predicate.

The analysis of the row2col/5 predicate based on the term-size norm will detect that the size of the third argument is less than or equal to that of the second argument. This is due to the base case for row $2 \mathrm{col} / 5$ where these two arguments are equal terms and hence also equal in term-size. An analyzer reasoning based on both term-size and list-length norms could detect in addition that the second and third arguments are equal in their term-size only when the list-length of the second argument is equal to zero and that in all other cases the inequality is strict. Given that the second argument in the call at program point (b) has a list-length greater than zero, the strict inequality is derived and termination can be proved.

However this is beyond the capabilities of analysers which consider single norms. We will revisit and solve this problem by combining the term-size and list-length norms using the techniques introduced in Section 4.

Third Observation: The transpose program terminates (in both directions) for matrices containing possibly non-ground elements:

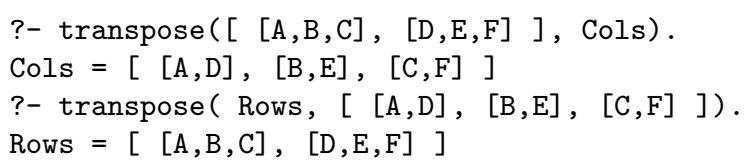

Here we also have a problem because using linear norms we cannot even say "list of list of possibly non-ground terms". This is because the cons function symbol occurs in two different contexts and must be considered differently in each context. At the top level we want to treat lists as does term-size, but at the inner level as does list-length. Such a distinction cannot be made using linear norms. For queries in which the first argument is given we can solve this problem using the more refined notion of type-based norms introduced in Section 3. For queries in which the second argument is given, we obtain a proof of termination by combining typebased norms hence taking advantage of the two techniques introduced in Sections 3 and 4 .

Fourth Observation: It is interesting to note that the transpose program terminates for queries which specify the number of rows and columns:

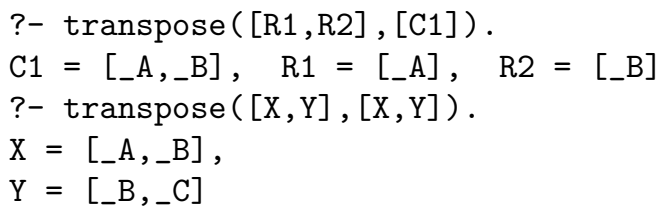

Termination for these types of queries can be shown using the list-length norm. For the one loop, at program point (C), the first argument is detected to be both decreasing and rigid, and for the other loop, at program point (d), the second argument is detected to be both decreasing and rigid. The size information for the list-length norm detected by the analyser is as follows:

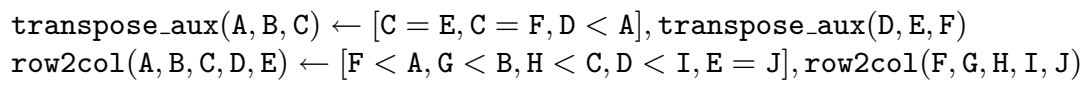


The contributions set forward in this paper address the deficiencies of current termination analysers for logic programs as demonstrated in the above examples.

\subsection{Type Information}

We consider a standard notion of types for logic programs [Somogyi et al. 1996]. Termination essentially has nothing to do with whether programs are typed or untyped. We use the types themselves just as a device to generate type-based norms; we introduce well-typed programs as a natural means of ensuring that type-based norms are applied consistently, and hence that abstraction based on type-based norms is sound.

Types, like terms are constructed from (type) variables and (type) symbols. We denote by $\mathcal{T}$ the set of types constructed from type variables $V_{\mathcal{T}}$ and type symbols $\Sigma_{\mathcal{T}}$. The sets of type variables, type symbols, variables and function symbols are assumed to be disjoint. A type containing variables is said to be polymorphic, otherwise it is monomorphic. In this paper we use only monomorphic types for termination analysis, but we introduce polymorphic types mainly because the notation is more convenient. For example, we can refer to the monomorphic types list(int) and list(list(int)) defined by instantiating the single definition of the polymorphic type list(T), rather than defining separate types.

Besides the usual substitutions which are mappings from variables to terms, we also have type substitutions which are mappings from type variables to types. For each type symbol, a unique type rule associates that symbol with a finite set of function symbols. We allow function symbols to be overloaded, i.e. they can be used in different type rules.

Definition 2.3. (type) $A$ type rule for a type symbol $h / n \in \Sigma_{\mathcal{T}}$ is of the form $h(\bar{v}) \longrightarrow f_{1}\left(\bar{\tau}_{1}\right) ; \ldots ; f_{k}\left(\bar{\tau}_{k}\right)$ where: $\bar{v}$ is an $n$-tuple of distinct type variables from $V_{\mathcal{T}}, f_{1}, \ldots, f_{k}$ are distinct function symbols from $\Sigma$ associated with the type symbol $h, \bar{\tau}_{i}(1 \leq i \leq k)$ are tuples from $\mathcal{T}$ of the appropriate arity, and type variables in $\bar{\tau}_{i}$, if any, are from $\bar{v}$. A type definition is a finite set of type rules for distinct type symbols. A type rule or type definition containing variables is said to be polymorphic, otherwise it is monomorphic.

The application of a type substitution to a type rule gives an instance of the original rule. A ground instance of a type rule, say $\left(h(\bar{v}) \longrightarrow f_{1}\left(\bar{\tau}_{1}\right) ; \ldots ; f_{k}\left(\bar{\tau}_{k}\right)\right) \theta$ determines the denotation of the type $h(\bar{v}) \theta$. In addition to types defined by the user, we allow also predefined types that have a predefined denotation. An example is the type int representing the set of integers.

EXAMPLE 2.5. Consider the following polymorphic type rules which define respectively lists and nested lists of elements of type $T$.

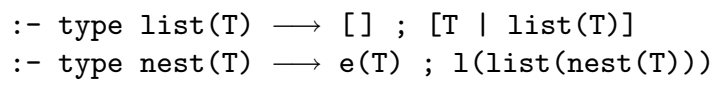

Below are instances which define the denotations of the following monomorphic types: list(list(int)) for $\mathrm{T}=$ list(int), list(int) for $\mathrm{T}=$ int, list(nest(int)) for $\mathrm{T}=\operatorname{nest}(\mathrm{int})$, and nest(int) for $\mathrm{T}=$ int. 


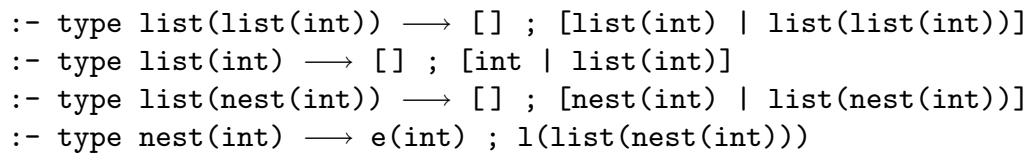

The denotation of list(list(int)) represents lists of lists of type int in which all subterms of type int are "nested at depth two". For example the terms [ ], [[1]], $[[1,2]]$ and $[[],[1,2]]$ all belong to the denotation of list(list(int)). The type nest(int) on the other hand, represents a data structure where elements of type int can be "nested" at any level. For example, the term e(3) contains an int at the top level, while $\mathrm{I}([\mathrm{e}(1), \mathrm{I}([\mathrm{e}(2), \mathrm{I}([\mathrm{e}(3), \mathrm{e}(4)])])])$ contains elements of type int at different levels. The functors e and 1 allow a parser to know whether the next subterm is either of type int or of type nest(int).

In what follows, we assume that programs are typed: it is assumed that the program contains a type definition $\rho$ and that each predicate $p / n$ has a unique declaration $p\left(\tau_{1}, \ldots, \tau_{n}\right)$ stating the (possibly polymorphic) types of its arguments. We assume that programs are well-typed and adopt a standard concept of a welltyping from [Mycroft and O'Keefe. 1984; Hill and Topor 1992].

To simplify the presentation of the concept of a well-typing, we assume for the moment that programs are in a normal form such that each atom is of the form: $p\left(X_{1}, \ldots, X_{n}\right), X=Y$ or $X=f\left(X_{1}, \ldots, X_{n}\right)$ (with $X_{1}, \ldots, X_{n}$ different variables). A variable typing is a function from variables to types. We say that a program $P$ has a well-typing if each clause $p\left(X_{1}, \ldots, X_{n}\right) \leftarrow B_{1}, \ldots, B_{m} \in P$ has a variable typing $\mu$ such that:

(1) $p\left(\mu\left(X_{1}\right), \ldots, \mu\left(X_{n}\right)\right)$ is the declared type of $p / n$;

(2) If $B_{i}$ is of the form $X=Y$ then $\mu(X)=\mu(Y)$;

(3) If $B_{i}$ is of the form $X=f\left(Y_{1}, \ldots, Y_{l}\right)$ then $\mu(X)=h(\bar{v}) \theta$ with $h$ a type symbol defined by a type rule

$$
h(\bar{v}) \longrightarrow \ldots ; f\left(\tau_{1}, \ldots, \tau_{m}\right) ; \ldots
$$

and $\mu\left(Y_{i}\right)=\tau_{i} \theta$ for each $i$; and

(4) If $B_{i}$ is of the form $q\left(Y_{1}, \ldots, Y_{l}\right)$ then $q\left(\mu\left(Y_{1}\right), \ldots, \mu\left(Y_{l}\right)\right)$ is an instance of the type declared for $q / l$.

The well-typing associates a type with each variable (and term) in the program. The notation $t: \tau$ is used to indicate that $\tau$ is the type of $t$. In the case of monomorphic types, a more restrictive version of Condition (4) applies.

(4') If $B_{i}$ is of the form $q\left(Y_{1}, \ldots, Y_{l}\right)$ then $q\left(\mu\left(Y_{1}\right), \ldots, \mu\left(Y_{l}\right)\right)$ is identical to the type declared for $q / l$.

If a well-typed program contains polymorphic predicates, multiple monomorphic versions can be generated for different uses of the predicate in order to obtain a monomorphically well-typed program satisfying Condition (4') : for example, separate procedures for appending lists of integers and for appending lists of names can be created. (In some cases an infinite number of type instances of a polymorphic predicate arises, when a polymorphic predicate calls some type instance of itself; if so, one option is to introduce a new monomorphic type to generalise 
an infinite number of types, for example list(any) to generalise types list(int), list(list(int)),....)

Generating several monomorphic instances of polymorphic predicates is a feasible approach, but in fact, termination of a query to a polymorphic predicate does not depend on the types $\tau_{i}$ that are the instances of the type parameters $T_{i}$. A polymorphic predicate can only pass around terms of type $T_{i}$ and can never construct or deconstruct them. Hence, one can consider the $\tau_{i}$ as anonymous monomorphic types. Although one can be interested in using the predicate for different instances of the type parameters, one termination proof, where the different type parameters are considered as distinct predefined types, suffices. This and other issues relating to termination analysis based directly on polymorphic types is the subject of ongoing research [Bruynooghe et al. 2002; Lagoon et al. 2003; Bruynooghe et al. 2003; Lagoon 2004].

The next definition specifies a notion of the constituents of a type. These are the possible types for sub-terms of terms of that type.

DEFINITION 2.4. (type constituent) Let $\tau$ and $\sigma$ be types. We say that $\sigma$ is a constituent of $\tau$, and denote $\sigma \preceq \tau$, if there exists a term of type $\tau$ which has a sub-term of type $\sigma$. The set of constituents of $\tau$ is denoted Constituents $(\tau)$. By slight abuse of notation, we denote by Constituents $(\rho)$ the union of the constituent sets for all types defined in a type definition $\rho$.

We adopt a common restriction in type-based program analysis, which is equivalent to requiring that Constituents $(\tau)$ is finite. This excludes type rules such as $t(\tau) \longrightarrow f(t(t(\tau)))$ which has an infinite set of constituents of the form $t^{n}(\tau)$ where $n \geq 1$.

EXAMPLE 2.6. In the context of Example 2.5, observe that:

- $\operatorname{Constituents}(T)=\{T\}$

- Constituents $($ int $)=\{$ int $\}$

- $\operatorname{Constituents}(\operatorname{list}(\operatorname{list}(T)))=\{T, \operatorname{list}(T), \operatorname{list}(\operatorname{list}(T))\}$

- Constituents $($ nest $($ int $))=\{$ int, nest $($ int $)$, list $($ nest $($ int $))\}$
- Constituents $($ list $(T))=\{T, \operatorname{list}(T)\}$

- Constituents $($ list $($ int $))=\{$ int, list $($ int $)\}$

Hence we have that int $\preceq$ int, int $\preceq l$ list(int), int $\preceq l$ list(nest(int)), etc.

\section{TYPE-BASED NORMS}

In this section we consider how norms can be defined based on type information. We refer to such norms as type-based norms. This problem has been considered previously in [Bossi et al. 1992; Decorte et al. 1993; 1996; Martin et al. 1996; Decorte et al. 1997]. Inferring norms from type information makes sense as recursive types represent recursive data-structures and thus identify potential sources of infinite recursion. Our approach is simple: for a type $\sigma$ we define a norm $\psi_{\sigma}$ which counts the number of non-variable sub-terms of type $\sigma$ in the term it is applied to. This means that the contribution of a functor depends on the type context in which it appears. This very simple idea differs from previous proposals and turns out to work well in practice, for example it solves the problem that a term cannot be measured differently depending on the context. 
Basing norms on type information has two advantages. First, it provides more refined size measures. This is because while (semi-) linear norms define the size of a term according to its prime functor (as a function of the size of its arguments), typebased norms define the size of a term based on its type context. This means that the same term can be measured differently depending on its type. This is particularly useful when the same function symbol may occur in different contexts as is the case for instance in Example 2.4. Second, it identifies a finite set of candidate norms for the analyser to consider - one for each type in the program. In practice, this set often includes norms isomorphic to those applied by the users of termination analysers.

As in the work on semi-linear norms we introduce two notions: the $\sigma$-size of a typed term $t$, denoted $t_{\sigma}$, which is a natural number; and its $\sigma$-abstraction, denoted $\mathrm{t}_{\sigma}^{\alpha}$, which is a linear expression containing size variables.

The following definition formalises the notion of $\sigma$-abstraction.

DEFINITION 3.1. (symbolic type-based abstraction) Let $\rho$ be a type definition, let $\sigma$ and $\tau$ be types predefined or defined in $\rho$, and let $c(\tau, \sigma)$ be a constant determined by $\sigma$ and $\tau$ such that $c(\tau, \sigma)$ equals to 1 if $\tau=\sigma$ and to 0 otherwise. The $\sigma$ abstraction of a term $t$ of type $\tau$ is denoted by $|t: \tau|_{\sigma}^{\alpha}$ and is computed by the following rules:

(1) If $t=f\left(t_{1}, \ldots, t_{n}\right)$ is of a defined type $\tau$ and $t_{1}, \ldots, t_{n}$ are respectively of type $\tau_{1}, \ldots, \tau_{n}$. Then there exists a rule $\tau \longrightarrow f_{1}\left(\bar{\tau}_{1}\right) ; \ldots ; f_{k}\left(\bar{\tau}_{k}\right)$ in $\rho$ and an $i \in\{1, \ldots, k\}$ such that $f\left(\tau_{1}, \ldots, \tau_{n}\right)=f_{i}\left(\bar{\tau}_{i}\right)$. We define:

$$
\left|t: \tau_{\sigma}^{\alpha}=c(\sigma, \tau)+\right| t_{1}:\left.\tau_{1}\right|_{\sigma} ^{\alpha}+\cdots+\left|t_{n}: \tau_{n}\right|_{\sigma}^{\alpha} .
$$

(2) If $t$ is a non-variable term of predefined type $\tau$, then $|t: \tau|_{\sigma}^{\alpha}=c(\sigma, \tau)$.

(3) Otherwise, $t$ is a variable, say $X$, and

$$
|t: \tau|_{\sigma}^{\alpha}= \begin{cases}c(\sigma, \tau)+X_{\sigma} & \text { if } \sigma \preceq \tau \\ 0 & \text { otherwise }\end{cases}
$$

Note in particular that for a variable $X$ of type $\tau$, if $\sigma=\tau$ then it is abstracted as $X_{\sigma}+1$. This is because any well-typed rigid instance of $X$ contains at least one sub-term of type $\sigma$. The explicit inclusion of the +1 ensures that $X_{\sigma}+1$ is strictly positive under the assumption that $X_{\sigma} \geq 0$, which is a uniform assumption on all size variables in the abstract program. Alternatively, we could have abstracted $X$ by $X_{\sigma}$ and added the constraint that $X_{\sigma}>0$ whenever $\sigma=\tau$, but this would be less convenient. In the following: (1) we drop the subscript $\sigma$ from $X_{\sigma}$ when no confusion can arise; (2) sometimes we write $e_{1}>e_{2}$ instead of writing $e_{1} \geq e_{2}+1$; and (3) we implicitly assume all size variables to be non-negative.

As any type has a finite number of constituents, Definition 3.1 implies that there is only a finite number of different ways to measure a term using type-based norms. Note that all type-based norms that do not correspond to one of the constituents of the term's type measure the term as zero.

EXAMPLE 3.1. The following table illustrates the $\sigma$-abstraction of several terms of type list(list(int)) for $\sigma \in\{$ int, list(int), list(list(int))\}. The $\sigma$-sizes for these terms are obtained by assigning the size variables in the abstractions to zero. 


\begin{tabular}{l||c|c|c}
\hline \hline$t: \operatorname{list}($ list $($ int $))$ & ||$_{\text {int }}^{\alpha}$ & $\left.\right|_{\text {list (int) }} ^{\alpha}$ & $\left.\right|_{\text {list (list (int }))} ^{\alpha}$ \\
\hline \hline[] & 0 & 0 & 1 \\
{$[[]]$} & 0 & 1 & 2 \\
{$[[1]]$} & 1 & 2 & 2 \\
{$[[1,2],[3]]$} & 3 & 5 & 3 \\
{$[[1,2],[X] \mid X s]$} & $2+\left(X_{\text {int }}+1\right)+X s_{\text {int }}$ & $5+X s_{\text {list (int })}$ & $2+\left(X s_{\text {list }(\text { list }(\text { int }))}+1\right)$
\end{tabular}

Note that sub-terms may be measured differently according to the type context in which they appear. When the term [] is considered to be of type list(list(int)) (as in the first row of the table), its list(int)-size is 0. If, on the other hand, [] is considered to be of type list(int) (as the innermost [] in the second row of the table), its list(int)-size is 1. Note also that the list(list(int))-size of the terms in the example is isomorphic to the list-length norm as the list-length of a term is one less than its list(list(int))-size.

Program abstraction with respect to a type-based norm $\left.\right|_{\sigma}$ is similar to the usual abstraction with respect to semi-linear norms: A term $t: \tau$ is replaced by $|t: \tau|_{\sigma}^{\alpha}$ and constraints are added to reflect the fact that all abstract variables are non-negative. Often, in the examples these constraints are implicit. The definition of type-based abstraction allows us to formulate a simple definition of $\sigma$-rigidity. The intuition behind this definition, is that a term is $\sigma$-rigid, if further instantiations cannot introduce new sub-terms of type $\sigma$.

DEFINITION 3.2. (type-based rigidity) With $\sigma$ a constituent of $\tau$, a term $t: \tau$ is $\sigma$-rigid iff for all variables $X: \tau^{\prime}$ occurring in $t, \sigma$ is not a constituent of $\tau^{\prime}$.

Rigidity information can be represented in the domain Pos of positive Boolean functions [Marriott and Søndergaard 1993]. A type-based rigidity analysis is developed in [Bruynooghe et al. 2001]. Note that the $\sigma$-size of a $\sigma$-rigid term $t: \tau$ is constant for all well-typed instances $t \theta$ of type $\tau$.

Definition 3.3. (type-based norm) The type-based norm $\|_{\sigma}$ is defined by assigning the value 0 to the variables in the $\sigma$-abstraction $\left\|_{\sigma}^{\alpha} \cdot\right\|_{\sigma}$ is also called the $\sigma$-size norm. The definition is such that $\phi_{\sigma}$ measures the minimal number of subterms of type $\sigma$ in the well-typed and $\sigma$-rigid instances of $t$.

EXAMPLE 3.2. Consider the append/3 predicate from Example 2.1 assuming the following type definition and declaration:

$:-$ type list(int) $\longrightarrow[] ;$ [int|list(int)] .

:- pred append(list(int), list(int), list(int)).

The int-and list(int)-abstractions are given below. The subscripts $i$ and $\ell(i)$ are used (as abbreviations) for int and list(int).

\begin{tabular}{|c|c|}
\hline list(int)-abstraction & int-abstraction \\
\hline $\begin{array}{l}\text { append }\left(1,1+Y \mathbf{s}_{\ell(i)}, 1+Y \mathbf{s}_{\ell(i)}\right) \\
\text { append }\left(2+X \mathbf{s}_{\ell(i)}, 1+Y \mathbf{s}_{\ell(i)}, 2+Z \mathbf{s}_{\ell(i)}\right):- \\
\quad \text { append }\left(1+X \mathbf{s}_{\ell(i)}, 1+Y \mathbf{s}_{\ell(i)}, 1+Z \mathbf{s}_{\ell(i)}\right) .\end{array}$ & $\begin{array}{l}\text { append }\left(0, Y \mathbf{s}_{i}, Y \mathbf{s}_{i}\right) . \\
\text { append }\left(\mathrm{X}_{i}+1+\mathrm{X} \mathbf{s}_{i}, Y \mathbf{s}_{i}, \mathrm{X}_{i}+1+\mathrm{Z} \mathbf{s}_{i}\right):- \\
\quad \text { append }\left(X \mathbf{s}_{i}, Y \mathbf{s}_{i}, Z \mathbf{s}_{i}\right) .\end{array}$ \\
\hline
\end{tabular}

ACM Transactions on Programming Languages and Systems, Vol. TBD, No. TDB, Month Year. 
The int-abstraction of the term [] is 0 reflecting the fact that it does not contain an integer value. Similarly, its list(int)-abstraction is 1 reflecting that it contains a single sub-term of type list(int). The list(int)-abstraction of $[\mathrm{X} \mid \mathrm{X} \mathbf{s}]$ is $2+\mathrm{X}_{\mathbf{s}_{\ell(i)}}$ since any well-typed $\mathrm{list(int)-rigid} \mathrm{instance} \mathrm{has} \mathrm{at} \mathrm{least} 2$ sub-terms of type list(int). Its int-abstraction, $1+\mathrm{X}_{i}+\mathrm{Xs}_{i}$ denotes the number of sub-terms of type int in any well-typed instance: $1+\mathrm{x}_{i}$ in the head and $\mathrm{Xs}_{i}$ in the tail. These abstractions are isomorphic to the list-length and term-size abstractions and both lead to a proof of termination.

The following example illustrates how type information can help to provide a finite number of candidate norms which can be tried one by one.

EXAMPLE 3.3. Consider again the program from Example 2.2 which constructs (bottom-up) parse trees for words in the context free language $a^{n} b^{n}$. We provide a type definition to represent the symbols that may occur in a sentential form. Recall that the non-terminals occur as terms representing partial parse trees. For the given grammar there are two terminals: "a" and "b", and a non-terminal "s" which can occur in a parse tree with either 2 or 3 children. The type definition:

$$
\text { :- type symbol } \longrightarrow \text { a;b;s(symbol, symbol); (symbol, symbol, symbol) }
$$

specifies the terminal and non-terminal symbols in the grammar and the type declarations for the predicates in the program are:

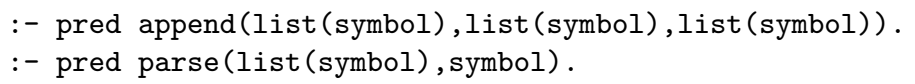

There are two type constituents: symbol and list(symbol). Termination analysis based on the symbol-abstraction fails to give a proof, similar to the use of term-size abstraction as described in Example 2.2. The list(symbol)-abstraction is isomorphic to the list-length abstraction, illustrated in Example 2.2 (the empty list [] is abstracted to 1 instead of to 0 ) and a proof of termination follows using this norm.

Basing norms on type information provides for more refined size and rigidity information. This is because unlike semi-linear norms the size of a term is computed according to its type and not according to its prime functor. This is particularly useful when the same function symbol is used to represent different data structures as for example when representing a matrix as a list of list of elements. In this case, when counting inner list symbols, a list of lists of variables is rigid.

EXAMPLE 3.4. Consider again the transpose/2 program from Example 2.4 with a query of the form transpose $([\mathrm{A}, \mathrm{B}, \mathrm{C}],[\mathrm{D}, \mathrm{E}, \mathrm{F}]], \mathrm{Col} \mathrm{s})$. We are not able to prove termination for such queries using semi-linear norms because we do not distinguish between the inner and outer list symbols in the matrix data structure. Using type-based norms we can do exactly that. Consider the norm which counts the number of inner list symbols in a list of lists: (1) In the loop at program point (c), the number of the inner list symbols in the first argument is both decreasing and rigid. It is decreasing because we remove the first row (i.e., $\mathrm{R}$ ) together with all of its inner lists (at least one) and it is rigid because if $[\mathrm{R} \mid \mathrm{Rs}]$ is rigid in its number of inner lists then so is Rs. (2) In the loop at program point (d), the number of the 
inner list symbols in the first argument decreases as we drop one element $(\mathrm{X})$ and it is rigid because if $[\mathrm{X} \mid \mathrm{Xs}]$ is rigid in the number of its list symbols then so is $\mathrm{Xs}$. To define the analysis based on this norm let us first add to the program suitable type declarations and definitions as follows (we take type int for the elements, but that could be any other type):

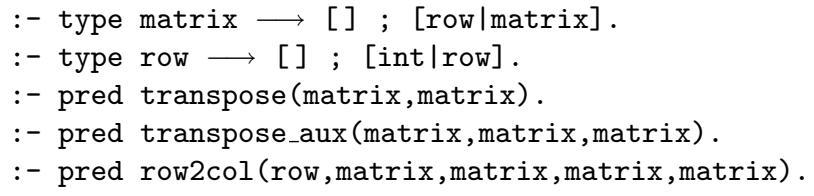

Here the type row represents the inner list symbols while the type matrix represents the outer lists. The row-abstraction of the program is:

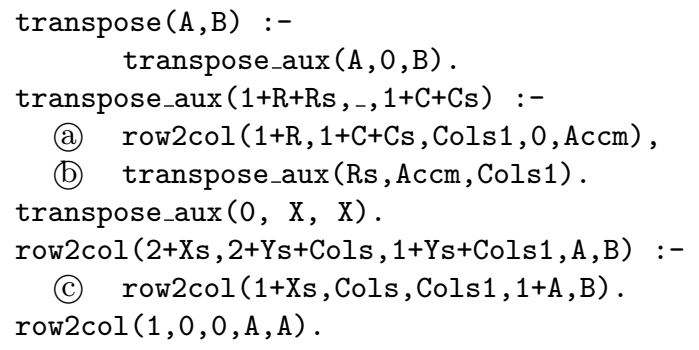

and this is exactly what we need to count the number of inner lists and prove termination. Note that we have omitted the constraints specifying the lower bounds on the values of the abstract variables.

From type-based norms to semi-linear norms. The correctness of our approach is presented based on the correctness of termination analysis using semi-linear norms as presented in Section 2. To this end, for a given well-typed program $P$ we construct a program $P^{\prime}$ (without types) with the same termination behaviour and show that the analysis of the well-typed program $P$ with respect to a type-based norm $\psi_{\sigma}$ is equivalent to the termination analysis of the untyped program $P^{\prime}$ with respect to a corresponding semi-linear norm which we denote as $|\cdot|_{s l(\sigma)}$. More precisely, the proof of correctness is organised in the following three steps:

(1) We define a mapping $\mathcal{U}$ from typed terms to untyped terms and apply it to transform $P$ to an untyped program $P^{\prime}$. We call $\mathcal{U}$ a term untyping.

(2) We show that $P$ and $P^{\prime}$ have the same termination behaviour. Namely $P$ terminates for a goal $G$ if and only if $P^{\prime}$ terminates for a corresponding query $G^{\prime}$ obtained by applying $\mathcal{U}$ to untype $G$.

(3) We define a semi-linear norm $\psi_{s l(\sigma)}$ such that the abstraction of a typed term $t: \tau$ with respect to $\psi_{\sigma}$ is identical up to variable renaming to the abstraction of its untyping $\mathcal{U}(t)$ with respect to $\left.\right|_{s l(\sigma)}$. The size abstractions for $P$ and $P^{\prime}$ with respect to $\psi_{\sigma}$ and $\psi_{s l(\sigma)}$ respectively are thus isomorphic. The rigidity abstractions for $P$ and $P^{\prime}$ are also isomorphic. Proposition 2.2 thus implies the correctness of termination analysis using type-based norms as stated in Theorem 3.1. 
Term untyping, as defined in the following definition, specifies the type of a term by a corresponding untyped term which contains special function symbols. The idea is to "wrap" each sub-term in the original term by a unique symbol which indicates its type.

DeFINITION 3.4. (Untyping) Let $\tau, \tau_{0}, \ldots$ be the types of the terms occurring in a well-typed syntactic object $P$. Let $f_{\tau}, f_{\tau_{0}}, \ldots$ be unary function symbols not occurring in $P$ corresponding to $\tau, \tau_{0}, \ldots$ respectively. The untyped syntactic object $P^{\mathcal{U}}$ corresponding to $P$ is obtained by untyping its terms. The untyping of a welltyped term $t: \tau$ is an untyped term defined (recursively) as follows:

$$
\mathcal{U}(t: \tau)= \begin{cases}f_{\tau}\left(f\left(\mathcal{U}\left(t_{1}\right), \ldots, \mathcal{U}\left(t_{n}\right)\right)\right) & \text { if } t=f\left(t_{1}, \ldots, t_{n}\right) \\ f_{\tau}(X) & \text { if } t=X \text { is a variable }\end{cases}
$$

EXAMPLE 3.5. (Untyping) Consider again the append/3 relation of Example 3.2. Let $\mathrm{li}$ and $\mathrm{i}$ be new unary function symbols corresponding to the types list(int) and int respectively. Then the following is the untyped version of append/3:

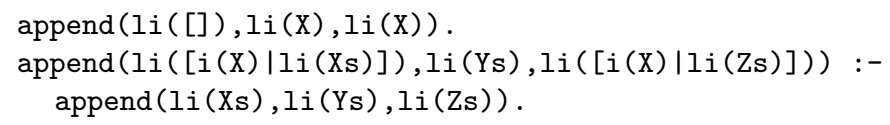

Lemma 3.1. For a given pair of well-typed terms $t_{1}: \tau$ and $t_{2}: \tau$, mgu $\left(t_{1}: \tau, t_{2}: \tau\right)$ exists if and only if $m g u\left(\mathcal{U}\left(t_{1}\right), \mathcal{U}\left(t_{2}\right)\right)$ exists.

Proof. Since the unification is between terms of the same type, the extra functor $f_{\tau}$ used to wrap terms of type $\tau$ has no influence on the result of the unification, hence success and failure of (an untyped version of) mgu are preserved.

Lemma 3.2. A well-typed query $G$ terminates for a well-typed program $P$ if and only if the corresponding query $\mathcal{U}(G)$ terminates for $\mathcal{U}(P)$.

ProOF. All unifications in LD-derivations for a well-typed goal and a well-typed program are between terms of the same type, and according to Lemma 3.1 two terms are unifiable if and only if the corresponding untyped terms are unifiable. Hence there is a 1-1 mapping between the LD-derivations for $G$ with $P$ and for $\mathcal{U}(G)$ with $\mathcal{U}(P)$.

We now define for a type-based norm ||$_{\sigma}$, its corresponding semi-linear norm on untyped terms. Recall that when defining a semi-linear norm the rules in the norm definition are always determined by the outermost function symbol of a term. In the definition, $f_{\tau} / 1$ denotes one of those fresh unary symbols indicating a type and $f / n$ indicates one of the original function symbols occurring in a term.

DEFINITION 3.5. (semi-linear version of symbolic type-based abstraction) Let $\sigma$ be a type, $\left.\right|_{\sigma}$ a type-based norm and $t$ an untyped term (the result of an untyping). We denote by ||$_{s l(\sigma)}^{\alpha}$ the abstraction with respect to the semi-linear norm 
corresponding to $\psi_{\sigma}$ :

$$
H_{s l(\sigma)}^{\alpha}= \begin{cases}c(\sigma, \tau)+\left|t^{\prime}\right|_{s l(\sigma)}^{\alpha} & \text { if } t=f_{\tau}\left(t^{\prime}\right) \text { and } \sigma \preceq \tau \\ \sum_{i=1}^{n}\left|t_{i}\right|_{s l(\sigma)}^{\alpha} & \text { if } t=f\left(t_{1}, \ldots, t_{n}\right) \\ X & \text { if } t=X \text { is a variable } \\ 0 & \text { otherwise }\end{cases}
$$

where $c(\tau, \sigma)$ is the same as in Definition 3.1.

EXAMPLE 3.6. The semi-linear version of the symbolic type-based abstraction $\|_{\sigma}^{\alpha}$ where $\sigma=\operatorname{list}($ int $)$ is:

$$
||_{s l(\sigma)}^{\alpha}= \begin{cases}1+\left|t^{\prime}\right|_{s l(\sigma)}^{\alpha} & \text { if } t=l i\left(t^{\prime}\right) \\ \left|t_{1}\right|_{s l(\sigma)}^{\alpha}+\left|t_{2}\right|_{s l(\sigma)}^{\alpha} & \text { if } t=\left[t_{1} \mid t_{2}\right] \\ X & \text { if } t=X \text { is a variable } \\ 0 & \text { otherwise }\end{cases}
$$

For example, the type-based list(int)-abstraction of $[X \mid Y]$ is $1+|X|_{l i}^{\alpha}+|Y|_{l i}^{\alpha}=$ $1+0+1+Y_{l i}=2+Y_{l i}$. The corresponding untyped term is $l i([i(X) \mid l i(Y)])$. Its abstraction according to the semi-linear sl(list(int)) norm is $1+\|[i(X) \mid l i(Y)]_{s l(l i)}^{\alpha}=$ $1+|i(X)|_{s l(l i)}^{\alpha}+|l i(Y)|_{s l(l i)}^{\alpha}=1+0+1+|Y|_{s l(l i)}^{\alpha}=2+Y_{s l(l i)}$.

Lemma 3.3. Let $\tau$ and $\sigma$ be types, $\|_{s l(\sigma)}$ the semi-linear norm corresponding to the type-based norm $\left.\right|_{\sigma}$ and t: $\tau$ a typed term. Then $\left.\mathcal{H}(t)\right|_{s l(\sigma)} ^{\alpha}$ and $|t: \tau|_{\sigma}^{\alpha}$ are identical up to variable renaming.

Proof. The proof is by structural induction on the term $t$. As a base case we consider variables and constants.

Base Case. Given a typed variable $X: \tau$, according to Definition 3.1 (item 3), the $\sigma$-abstraction of $X: \tau$ is $\mid X: \tau_{\sigma}^{\alpha}=c(\sigma, \tau)+X$. The untyping of $X: \tau$ is $f_{\tau}(X)$ and according to Definition 3.5 its $\sigma$-abstraction is $\left|f_{\tau}(X)\right|_{s l(\sigma)}^{\alpha}=c(\sigma, \tau)+X$ which is equivalent (up to variable renaming) to $\mid X: \tau_{\sigma}^{\alpha}$. Similarly, the $\sigma$-abstractions of a constant a: $\tau$ and its untyped term $f_{\tau}(a)$ are respectively $|a:|_{\sigma}^{\alpha}=c(\sigma, \tau)$ and $\left|f_{\tau}(a)\right|_{s l(\sigma)}^{\alpha}=c(\sigma, \tau)$.

Induction Step: Suppose the Lemma holds for all well-typed terms of depth smaller than $n$, we will show that it holds also for terms of depth $n$. Let $f\left(t_{1}, \ldots, t_{k}\right): \tau$ be a well-typed term of depth $n$. According to Definition 3.1 its $\sigma$-abstraction is $\left|f\left(t_{1}, \ldots, t_{k}\right): \tau\right|_{\sigma}^{\alpha}=c(\sigma, \tau)+\left|t_{1}\right|_{\sigma}^{\alpha}+\ldots+\left|t_{k}\right|_{\sigma}^{\alpha}$. The untyping of $f\left(t_{1}, \ldots, t_{k}\right): \tau$ is the term $f_{\tau}\left(f\left(\mathcal{U}\left(t_{1}\right), \ldots, \mathcal{U}\left(t_{k}\right)\right)\right)$, and according to Definition 3.5 its abstraction is $\left|f_{\tau}\left(f\left(t_{1}, \ldots, t_{k}\right)\right)\right|_{s l(\sigma)}^{\alpha}=c(\sigma, \tau)+\left.\mathcal{M}\left(t_{1}\right)\right|_{s l(\sigma)} ^{\alpha}+\ldots+\left.\mathcal{M}\left(t_{k}\right)\right|_{s l(\sigma)} ^{\alpha}$. By inACM Transactions on Programming Languages and Systems, Vol. TBD, No. TDB, Month Year. 
duction hypothesis, $\left|t_{i}\right|_{\sigma}^{\alpha}=\left.\mathcal{H}\left(t_{i}\right)\right|_{s l(\sigma)} ^{\alpha}$, hence the expressions $\left|f\left(t_{1}, \ldots, t_{k}\right)\right|_{\sigma}^{\alpha}$ and $\mid f_{\tau}\left(f\left(\mathcal{U}\left(t_{1}\right), \ldots, \mathcal{U}\left(t_{k}\right)\right)\right): \tau_{s l(\sigma)}^{\alpha}$ are equivalent up to variable renaming.

The correctness of type-based termination analysis is thus established, by showing (using Lemmata 3.2 and 3.3) that the abstraction of the typed program using type-based norms is semantically equivalent to the abstraction of the corresponding untyped program and semi-linear versions of the type-based norms. As elaborated on in Section 2.1, termination analysis based on semi-linear norms is known to be correct.

TheOREM 3.1. (correctness of type-based termination) Let $P$ be a program, and $H_{\sigma}$ a type-based norm and $G^{a}$ an initial call pattern specifying the arguments in the initial query which are rigid with respect to $H_{\sigma}$. Assuming the conditions of Proposition 2.2, $P$ terminates for all initial queries described by $G^{a}$.

One might observe that the term untyping of a program is simply a renaming of a function symbol that occurs in different type contexts. A similar technique is often applied manually by the users of a termination analyser based on semi-linear norms. For example using two distinct list constructors, one for outer and one for inner lists, to represent a matrix. This manual process is error prone and one must take care to rename function symbols consistently. Typing the program makes the renaming unnecessary. The untyping transformation can be viewed as a way to perform a consistent renaming.

\section{COMBINING NORMS}

There are several reasons to consider a combination of different norms when analysing the termination behaviour of a given program. First, it can be the case that different norms are required in the proofs of termination for different components of the program. Second, in some cases it is more natural to express the required norm for a proof in terms of several simpler norms. Finally, as we show in this section, dependencies between different norms can improve the precision of the termination analysis.

One of the main problems encountered already in previous work [Decorte et al. $1996 ; 1997]$ concerns the interaction between size and rigidity information of program variables with respect to different norms. In terms of abstract compilation, variables in an abstracted program range over information about size and rigidity with respect to a given norm. One must know how to interpret values for each abstracted variable - with respect to which norm; and one must take care that variables abstracted by different norms do not interfere - if different occurrences of variable $X$ are abstracted by different norms then this will lead to problems. In our approach, these problems are avoided by separating the size information with respect to the different norms. In terms of program abstraction, the abstraction of a program variable $X$ with respect to different norms gives different abstract variables. E.g., the term-size and list-length abstractions of variable $X$ are $X_{t s}$ and $X_{l l}$ respectively. Likewise, the int- and list(int)-abstractions of the typed variable $X: l i s t(i n t)$ are $X_{\text {int }}$ and $X_{\text {list }(i n t)}$.

This section introduces a simple technique which enables a termination analyser to consider a combination of several norms. The key idea in our contribution is 
to combine norms by applying them simultaneously. This means that each argument in a predicate of the original program is replaced by several (renamed apart) abstract arguments each one specifying size and rigidity information with respect to the corresponding norm. This technique is extremely easy to implement. The advantage is that inter-argument size relations can provide information about the dependencies with respect to each norm and between different norms.

The novelty in our overall approach is to apply this idea in combination with the technique described in Section 3. Namely, (1) the types in a program suggest a collection of type-based norms; and (2) that collection is the basis for applying a single combined analysis. Experimental evaluation, as presented in Section 5, indicates that this works well in practice for a wide range of programs. We introduce the technique and its advantage through a series of examples and then argue its correctness.

EXAMPLE 4.1. Consider the following program where some of the program points have been annotated (e.g., (a).

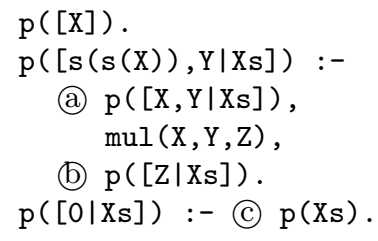

We omit the definition of $\mathrm{mul}(\mathrm{X}, \mathrm{Y}, \mathrm{Z})$, which defines the relation " $\mathrm{Z}$ is the multiplication of $\mathrm{X}$ and $\mathrm{Y}$ ", and concentrate only on the loops of $\mathrm{p} / 1$. The program is contrived to contain three different types of loops: (1) those where the list-length is invariant but the term-size decreases - recursive calls to point (a), (2) those where list-length decreases but term-size may increase - recursive calls to point (b), and (3) those where both measures decrease - recursive calls to point (c). There are additional loops resulting from the composition of these three. The program terminates for ground queries. It seems natural to consider the term-size and list-length norms to prove termination for this example. However, termination analysis, based on monotonicity constraints, using either one of these single norms does not succeed. The abstractions of the program with respect to these two norms are given below (left and middle). For the term-size abstraction, the loop at program point (b) cannot be shown to be decreasing and for the list-length abstraction, the loop at program point (a) cannot be shown to be decreasing. The combined abstraction with respect to the two norms together is also given below (right). Note that for the combined analysis the size variables have been renamed apart.

\begin{tabular}{c|c|c} 
term-size abstraction & list-length abstr. & combined abstraction \\
\hline $\mathrm{p}(2+\mathrm{X})$. & $\mathrm{p}(1)$. & $\mathrm{p}\left(2+\mathrm{X}_{t s}, 1\right)$. \\
$\mathrm{p}(4+\mathrm{X}+\mathrm{Y}+\mathrm{X} \mathrm{s}):-$ & $\mathrm{p}(2+\mathrm{Xs}):-$ & $\mathrm{p}\left(4+\mathrm{X}_{t s}+\mathrm{Y}_{t s}+\mathrm{X} \mathbf{s}_{t s}, 2+\mathrm{X} \mathbf{s}_{l l}\right):-$ \\
(a) $\mathrm{p}(2+\mathrm{X}+\mathrm{Y}+\mathrm{X} \mathrm{s})$, & (a) $\mathrm{p}(2+\mathrm{Xs})$, & $\mathrm{p}\left(2+\mathrm{X}_{t s}+\mathrm{Y}_{t s}+\mathrm{X} \mathbf{s}_{t s}, 2+\mathrm{X} \mathbf{s}_{l l}\right)$, \\
$\mathrm{mul}(\mathrm{X}, \mathrm{Y}, \mathrm{Z})$, & $\mathrm{mul}(\mathrm{X}, \mathrm{Y}, \mathrm{Z})$, & $\mathrm{mul}\left(\mathrm{X}_{t s}, \mathrm{X}_{l l}, \mathrm{Y}_{t s}, \mathrm{Y}_{l l}, \mathrm{Z}_{t s}, \mathrm{Z}_{l l}\right)$, \\
(b) $\mathrm{p}(1+\mathrm{Z}+\mathrm{Xs})$. & (b) $\mathrm{p}(1+\mathrm{Xs})$. & $\mathrm{p}\left(1+\mathrm{Z}_{t s}+\mathrm{X} \mathbf{s}_{t s}, 1+\mathrm{X} \mathbf{s}_{l l}\right)$. \\
$\mathrm{p}(2+\mathrm{Xs}):-$ & $\mathrm{p}(1+\mathrm{Xs}):-$ & $\mathrm{p}\left(2+\mathrm{X} \mathbf{s}_{t s}, 1+\mathrm{X} \mathbf{s}_{l l}\right):-$ \\
(c) $\mathrm{p}(\mathrm{Xs})$. & (C) $\mathrm{p}(\mathrm{Xs})$. & $\mathrm{p}\left(\mathrm{X}_{t s}, \mathrm{X} \mathbf{s}_{l l}\right)$.
\end{tabular}

ACM Transactions on Programming Languages and Systems, Vol. TBD, No. TDB, Month Year. 
The following loops are detected by the termination analyser when considering the combined norms:

$$
\begin{aligned}
& \mathrm{C} 1: \mathrm{p}\left(\mathrm{X}_{t s}, \mathrm{X}_{l l}\right) \leftarrow\left[\mathrm{X}_{l l}=\mathrm{X}_{l l}^{\prime}, \mathrm{x}_{t s}^{\prime}<\mathrm{X}_{t s}\right], \mathrm{p}\left(\mathrm{x}_{t s}^{\prime}, \mathrm{x}_{l l}^{\prime}\right) . \\
& \mathrm{C} 2: \mathrm{p}\left(\mathrm{X}_{t s}, \mathrm{X}_{l l}\right) \leftarrow\left[\mathrm{x}_{l l}^{\prime}<\mathrm{X}_{t s}, \mathrm{x}_{l l}^{\prime}<\mathrm{X}_{l l}\right], \mathrm{p}\left(\mathrm{X}_{t s}^{\prime}, \mathrm{x}_{l l}^{\prime}\right) \text {. } \\
& \text { C3: } \mathrm{p}\left(\mathrm{X}_{t s}, \mathrm{X}_{l l}\right) \leftarrow\left[\mathrm{X}_{t s}^{\prime}<\mathrm{X}_{t s}, \mathrm{x}_{l l}^{\prime}<\mathrm{X}_{l l}\right], \mathrm{p}\left(\mathrm{X}_{t s}^{\prime}, \mathrm{x}_{l l}^{\prime}\right) \text {. }
\end{aligned}
$$

The analysis determines that all loops decrease in at least one of the measures and gives sufficient information concerning rigidity. Hence termination is guaranteed. Note that this information cannot be recovered from separated term-size and listlength analyses.

This program can be typed by adding the following declaration and definitions:

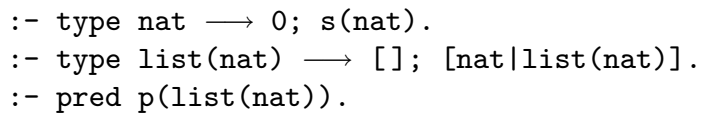

The combined analysis based on the two type constituents, nat and list(nat), determines termination and is very similar to the combined term-size and listlength analysis.

The previous example highlights an important issue. The analyser identifies all of the loops in the program and characterises each loop by size and rigidity information. To prove termination it is sufficient to show that each of the program loops "terminates" with respect to one of the norms involved. This is related to the fact that the abstraction introduces additional arguments for each of the norms. It boils down to choosing a different level mapping for each abstract binary clause. In the combined analysis, different level mappings might focus on arguments originating from the same norm or from a collection of different norms.

The next example is not contrived. The program is a classic in the area of termination analysis. It specifies (some of the) rules for computing the repeated differentiation of a function. Folklore has it that Floyd gave this program as a problem on a Ph.D. qualifying exam at Carnegie-Mellon in 1967 expecting the students to use ordinals to solve it. In our context, the level mapping required to prove termination is non-linear (see [Dershowitz and Manna 1979]). It has been reported [Serebrenik and De Schreye 2001] that no automatic system for proving termination on the basis of level mappings is able to generate mappings of the type required to prove termination for this example. We illustrate a proof of termination based on a combination of two simple norms derived automatically from the type definitions associated with this program.

ExAmPle 4.2. The following Prolog program (below right), quoted from [Serebrenik and De Schreye 2001], formulates some of the rules for computing the repeated differentiation of a function. The function is an expression where $\mathrm{t}$ is a variable and d stands for "derivative of". The original statement of the problem is given in terms of rewrite systems. We quote it (below left) from [Dershowitz 1995] (for conciseness we omit the rule for subtraction). Note that the Prolog version is slightly deficient as for example indicated by the query $\mathrm{p}(\mathrm{d}(\mathrm{d}(\mathrm{t}) * \mathrm{~d}(\mathrm{t}))$, Ans) which gives the answer $\mathrm{Ans}=\mathrm{d}(\mathrm{t}) * 0+\mathrm{d}(\mathrm{t}) * 0$. Using the rewrite system we always can get an expression without derivation symbols. The Prolog version does work as expected when applied to compute repeated derivations of the function in the single 
variable $\mathrm{t}$. This however is not our concern. The program in this form is considered to pose a challenge for termination analysis of logic programs.

\begin{tabular}{l|c} 
rewriting & Prolog \\
\hline & $\mathrm{p}(\mathrm{d}(\mathrm{t}), 1)$. \\
$\mathrm{p}(\mathrm{d}(\mathrm{A}), \mathrm{O}):-\operatorname{number}(\mathrm{A})$. \\
$\mathrm{D} \mathrm{t} \rightarrow \mathrm{p}(\mathrm{d}(\mathrm{X}+\mathrm{Y}), \mathrm{DX}+\mathrm{DY}):-$ \\
$\mathrm{D}(\mathrm{constant}) \rightarrow 0$ & $\mathrm{p}(\mathrm{d}(\mathrm{X}), \mathrm{DX}), \mathrm{p}(\mathrm{d}(\mathrm{Y}), \mathrm{DY})$. \\
$\mathrm{D}(\mathrm{x}+\mathrm{y}) \rightarrow \mathrm{D} \mathrm{x}+\mathrm{D} \mathrm{y}$ & $\mathrm{p}(\mathrm{d}(\mathrm{X} * \mathrm{Y}), \mathrm{X} * \mathrm{DY}+\mathrm{Y} * \mathrm{DX}):-$ \\
$\mathrm{D}(\mathrm{x} * \mathrm{y}) \rightarrow(\mathrm{y} * \mathrm{D} \mathrm{x})+(\mathrm{x} * \mathrm{D} \mathrm{y})$ & $\mathrm{p}(\mathrm{d}(\mathrm{X}), \mathrm{DX}), \mathrm{p}(\mathrm{d}(\mathrm{Y}), \mathrm{DY})$. \\
& $\mathrm{p}(\mathrm{d}(\mathrm{d}(\mathrm{X})), \mathrm{DDX}):-$ \\
& $\mathrm{p}(\mathrm{d}(\mathrm{X}), \mathrm{DX}), \mathrm{p}(\mathrm{d}(\mathrm{DX}), \mathrm{DDX})$.
\end{tabular}

We show that after typing the Prolog program our system can prove its termination automatically. Moreover, typing the program restricts it to the inputs for which it works correctly. We distinguish between two types of terms in the program: expressions in the single variable $t$ and derivation tasks. Expressions are specified by the type definition:

$:-$ type $\exp \longrightarrow t ;$ const (int); +(exp, exp); *(exp,exp).

Note that the syntax for types requires that we "wrap" the (predefined) type int on the right side of a rule with a function symbol as type rules are of the form $\mathrm{h}(\overline{\mathrm{v}}) \longrightarrow \mathrm{f}_{1}\left(\bar{\tau}_{1}\right) ; \ldots ; \mathrm{f}_{\mathrm{k}}\left(\bar{\tau}_{\mathrm{k}}\right)$. Derivation tasks are specified by the type definition:

$$
:- \text { type dt } \longrightarrow \mathrm{d}(\mathrm{dt}) ; \mathrm{e}(\exp ) \text {. }
$$

Here too we have wrapped the types occurring on the right side of a rule with a function symbol. The typed program (which includes the wrapper symbols) is:

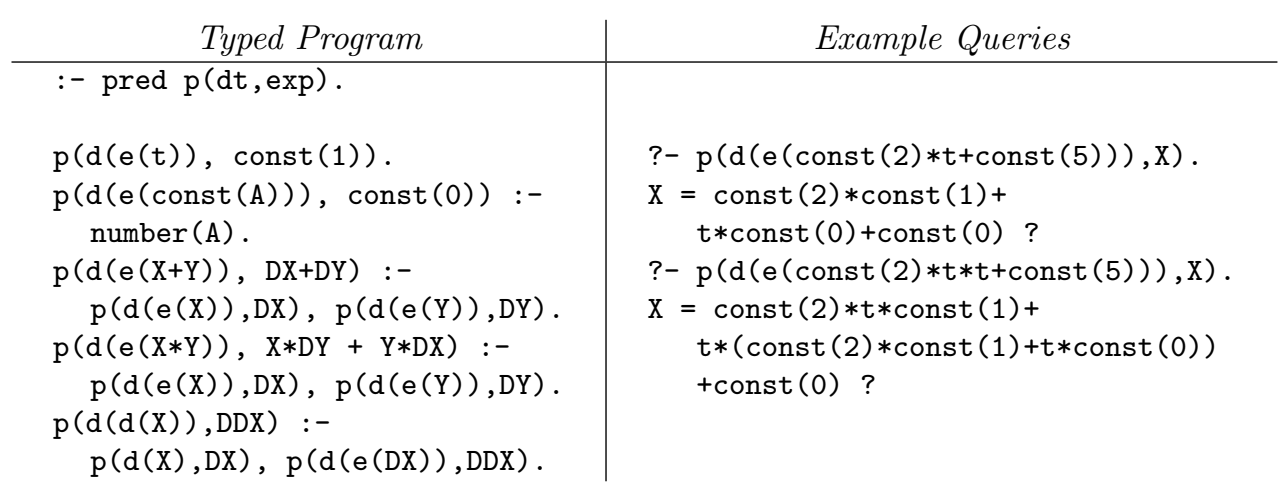

There are two type constituents to consider in the termination analysis. The corresponding norms count respectively the number of expression symbols, and the number of derivation tasks in a term. The analysis detects two forms of loops. For simplicity we show here only the constraints concerning the dt-and exp-sizes of the first argument:

$$
\begin{aligned}
& \mathrm{p}\left(\mathrm{D}_{d t}, \mathrm{D}_{\text {exp }}, \mathrm{E}_{d t}, \mathrm{E}_{\text {exp }}\right) \leftarrow\left[\mathrm{D}_{d t}=\mathrm{D}_{d t}^{\prime}, \mathrm{D}_{\text {exp }}^{\prime}<\mathrm{D}_{\text {exp }}, \ldots\right], \quad \mathrm{p}\left(\mathrm{D}_{d t}^{\prime}, \mathrm{D}^{\prime}{ }_{\text {exp }}, \mathrm{E}_{d t}^{\prime}, \mathrm{E}^{\prime}{ }_{e x p}\right) . \\
& \mathrm{p}\left(\mathrm{D}_{d t}, \mathrm{D}_{\text {exp }}, \mathrm{E}_{d t}, \mathrm{E}_{\text {exp }}\right) \leftarrow\left[\mathrm{D}_{d t}^{\prime}<\mathrm{D}_{d t}, \ldots\right], \quad \mathrm{p}\left(\mathrm{D}_{d t}^{\prime}, \mathrm{D}^{\prime}{ }_{\text {exp }}, \mathrm{E}_{d t}^{\prime}, \mathrm{E}_{\text {exp }}^{\prime}\right) .
\end{aligned}
$$

This together with the associated rigidity information is sufficient to prove termination for queries in which the first argument is a ground derivation task. 
The next example illustrates some additional advantages of combining norms. Here the recursion is not direct and the interaction between the different norms improves the quality of the inter-argument size relations derived.

EXAMPLE 4.3. Consider again the program from Example 2.4 which transposes the elements in a matrix and consider a query in which the second argument is a list of list of ground terms. We have already noted in Example 2.4 that we cannot prove termination for such queries using the term-size norm, nor using the list-length norm. The key to proving termination for this example is to detect that there is a strict decrease in size of the third argument in the recursive clause:

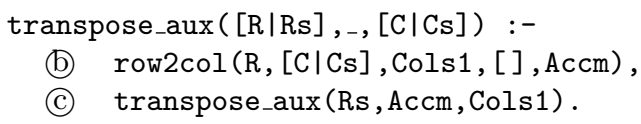

The relation between the sizes of the terms [C|Cs] in the head and Cols1 in the recursive call at program point (c) is determined by the occurrences of these terms in the call to row2col at program point (b). So we focus on the sizes of the second and third arguments in this predicate:

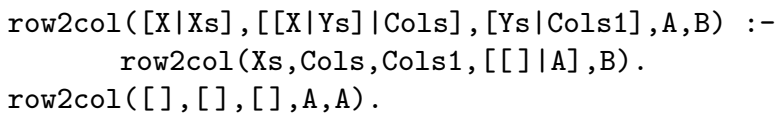

If we consider the seperate list-length and term-size analyses we do not detect a strict inequality between the sizes of these arguments. The results obtained are of the form:

$$
\begin{aligned}
& \operatorname{row} 2 \operatorname{col}\left(\mathrm{A}_{l l}, \mathrm{~B}_{l l}, \mathrm{C}_{l l}, \mathrm{D}_{l l}, \mathrm{E}_{l l}\right):-\left[\mathrm{B}_{l l}=\mathrm{C}_{l l}, \ldots\right] \\
& \operatorname{row} 2 \operatorname{col}\left(\mathrm{A}_{t s}, \mathrm{~B}_{t s}, \mathrm{C}_{t s}, \mathrm{D}_{t s}, \mathrm{E}_{t s}\right):-\left[\mathrm{B}_{t s} \geq \mathrm{C}_{t s}, \ldots\right]
\end{aligned}
$$

However considering the combined term-size and list-length abstraction (and focusing only on the second and third arguments):

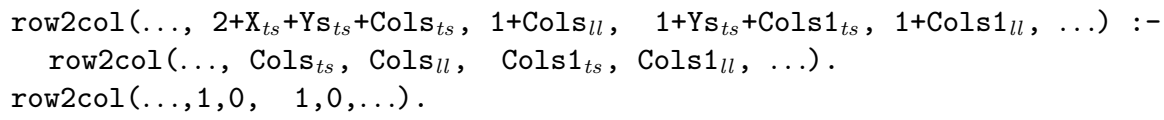

Here we obtain a result of the form:

$\operatorname{row} 2 \operatorname{col}\left(\ldots, \mathrm{B}_{t s}, \mathrm{~B}_{l l}, \mathrm{C}_{t s}, \mathrm{C}_{l l}, \ldots\right):-\left[\mathrm{B}_{l l}=\mathrm{C}_{l l}, \mathrm{~B}_{t s} \geq \mathrm{C}_{t s}+\mathrm{B}_{l l}, \ldots\right]$

Hence, for any call to row2col in which the second argument is a non-empty list (and so $\mathrm{B}_{l l}>0$ ) we will have the strict inequality $\mathrm{B}_{t s}>\mathrm{C}_{t s}$. Observe that the call at program point (b) has a non-empty list as a second argument. Note that a similar proof can be obtained for the combination of the type-based norms $\|_{\text {row }}$ and $\psi_{\text {matrix }}$.

Finally, let us consider another classic example, termination of which cannot be shown automatically using existing systems.

EXAMPLE 4.4. Consider the following program (14.4 in [Sterling and Shapiro 1994]) which colours a map so that no two adjacent regions have the same colour. The predicates member $/ 2$ and select $/ 3$ (omitted) are standard predicates (see [Sterling and Shapiro 19941). A map is represented as a list of regions where each region has a colour and a list of colours for the adjoining regions. An example initial query 
is given in the box: note that in the initial query the colours of the regions are given by means of free logic variables, since the program is going to decide which colour is associated with which region in order to avoid colouring two adjacent regions with the same colour (note the use of shared variables). The type declarations and definitions are given as:

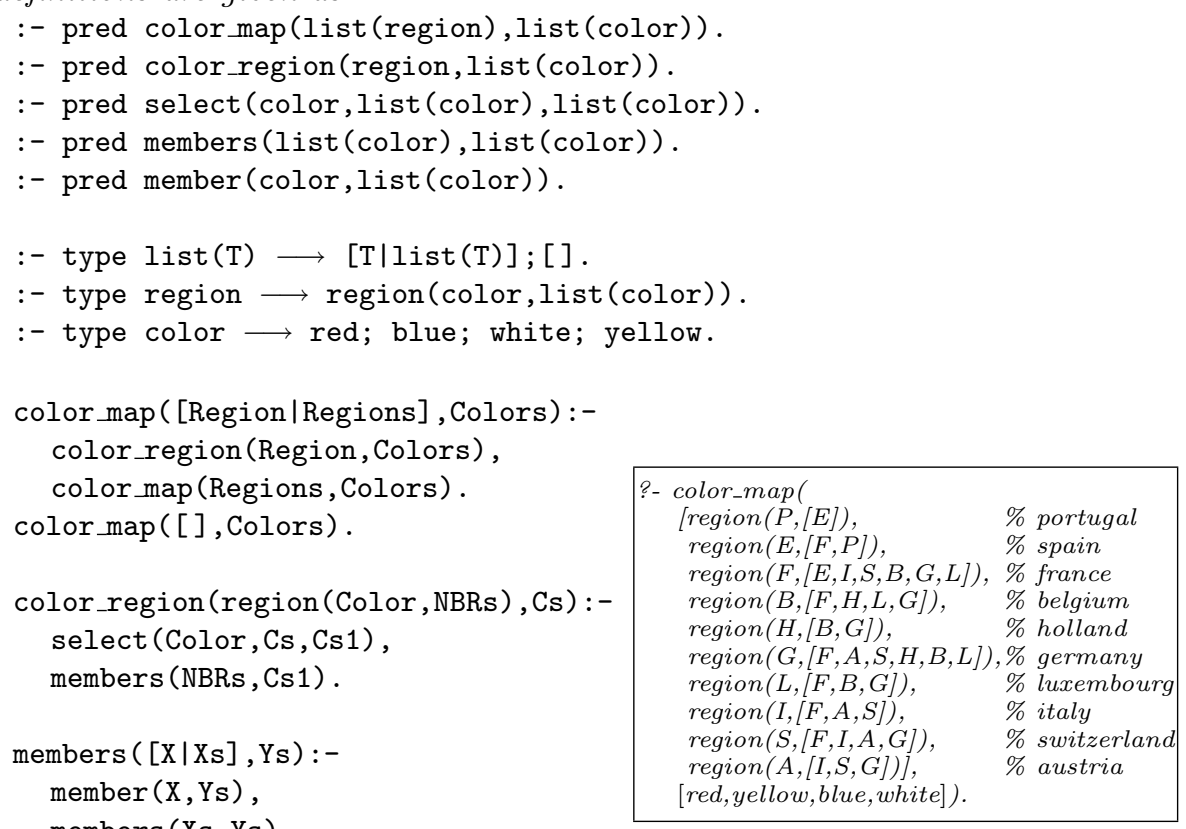

Proving termination for this program is not straightforward and cannot be performed using the available termination analysers. The term-size norm is not suitable because the list of regions in the initial query contains variables (and hence is not rigid with respect to term-size). The list-length norm is not suitable because the first clause invokes a call to color_region/2 which traverses the list of neighbours in that region (so even though the first argument in color_map/2 is expected to be a rigid list, all information about the elements of that list is ignored and in particular, the ability to reason about the rigidity of the lists of neighbours inside these elements is lost). A specialised semi-linear norm cannot be defined because the list functor occurs in two different type contexts (for regions and for neighbours) and should be treated differently in each. Using norms derived from types we obtain a suitable combined norm and a proof of termination.

From multiple norms to a single norm. To justify the correctness of our approach we show that our combined termination analysis of a program $P$ with respect to a collection $\mathcal{N}$ of norms (semi-linear or type-based) is equivalent to the termination analysis of another program $P^{\mathcal{N}}$ which has the same termination behaviour as $P$ with a single norm ||$_{\mathcal{N}}$. Here, Equivalent means that when we abstract $P^{\mathcal{N}}$ with respect to the single norm we obtain exactly the same abstract program as when we perform our combined analysis for $P$ with the combination of norms. Hence the correctness of termination analysis of a program using one of these approaches is 
the same as using the other. In the rest of this section we assume given a program $P$ and a collection of $k$ norms $\mathcal{N}=\left\{|\cdot|_{1}, \ldots,||_{k}\right\}$.

Step 1: To obtain $P^{\mathcal{N}}$ we "duplicate" the program $P$ taking $k$ copies, each containing different function symbols and variable names. We combine these copies into a single program putting them side by side, replacing each $n$-ary relation by a corresponding $(k * n)$-ary relation. For example, duplicating two times the third clause of the $p / 2$ predicate of Example 4.2 yields the following clause:

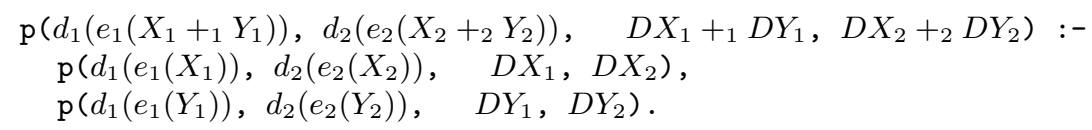

Renaming the function symbols enables us, in the next step, to consider renaming of the norms in such a way that they will not interact during program abstraction. The termination behaviour of the duplicated program is the same as that of the original - for corresponding queries. Intuitively, the execution of a query $G^{\mathcal{N}}$ with respect to $P^{\mathcal{N}}$ is like executing $k$ copies of $G$ with respect to $P$ simultaneously since the different copies do not share function symbols or variables. To prove (formally) this claim, we introduce a term renaming operator and then proceed to $k$-duplicate the atoms in $P$.

Definition 4.1. (Term renaming) A term renaming operator is a mapping $\Delta^{i}$ : Terms $\mapsto$ Terms and defined as follows:

$$
\Delta^{i}(t)= \begin{cases}f_{i}\left(\Delta^{i}\left(t_{1}\right), \ldots, \Delta^{i}\left(t_{n}\right)\right) & \text { if } t=f\left(t_{1}, \ldots, t_{n}\right) \\ X_{i} & \text { if } t \text { is a variable } X\end{cases}
$$

Example 4.5. Let $t=d(e(X+Y))$ and let $\Delta^{1}$ be a renaming operator, then $\Delta^{1}(t)=d_{1}\left(e_{1}\left(X_{1}+{ }_{1} Y_{1}\right)\right)$.

DeFinition 4.2. (k-duplication) Given an $n$-ary atom $A=p\left(t_{1}, \ldots, t_{n}\right)$ and an integer $k$ the $k$-duplication of $A$ is an $n * k$-ary atom

$$
A^{k}=p\left(\Delta^{1}\left(t_{1}\right), \ldots, \Delta^{k}\left(t_{1}\right), \ldots, \Delta^{1}\left(t_{n}\right), \ldots, \Delta^{k}\left(t_{n}\right)\right) .
$$

For $\mathcal{N}=\left\{||_{1}, \ldots,||_{k}\right\}$, we let $A^{\mathcal{N}}$ denote the $k$-duplication of atom $A$. For a program $P$ we let $P^{\mathcal{N}}$ denote the $k$ duplication of $P$ which is obtained by $k$-duplicating its atoms.

Example 4.6. Let $t=d(e(X+Y)), t^{\prime}=D X+D Y$, and $A=p\left(t, t^{\prime}\right)$ then:

$$
A^{2}=p(\underbrace{d_{1}\left(e_{1}\left(X_{1}+Y_{1}\right)\right)}_{\Delta^{1}(t)}, \underbrace{d_{2}\left(e_{2}\left(X_{2}+Y_{2}\right)\right)}_{\Delta^{2}(t)}, \underbrace{D X_{1}+{ }_{1} D Y_{1}}_{\Delta^{1}\left(t^{\prime}\right)}, \underbrace{D X_{2}+{ }_{2} D Y_{2}}_{\Delta^{2}\left(t^{\prime}\right)})
$$

Proposition 4.1. Let $G$ be an initial query, $P$ a logic program and $\mathcal{N}$ a collection of norms. Then, $P$ terminates for $G$ if and only if $P^{\mathcal{N}}$ terminates for $G^{\mathcal{N}}$.

Proof. Since the $k$ parts of each clause in $P^{\mathcal{N}}$ do not share function symbols and variables, a step $A \stackrel{\theta}{\sim} B$ in the $L D$ derivation of $G$ and $P$ corresponds to the step $A^{k} \stackrel{\theta^{\prime}}{\sim} B^{k}$ in the LD derivation of $G^{\mathcal{N}}$ and $P^{\mathcal{N}}$ where $\theta^{\prime}=\Delta^{1}\left(\theta_{1}\right) \cup \ldots \cup \Delta^{k}\left(\theta_{k}\right)$, and hence the $L D$ derivations are isomorphic. 
Now we can proceed to the next step which defines a single norm $\left.\right|_{\mathcal{N}}$ such that the abstraction of $P^{\mathcal{N}}$ with respect to this norm is identical (up to variable renaming) to the combined abstraction of $P$ with respect to the norms in $\mathcal{N}$.

Step 2:. To obtain a single norm $\|_{\mathcal{N}}$ we rename and duplicate the symbols occurring in the norms in $\mathcal{N}$ to be consistent with the term renamings in the duplicated program. We first note that the notion of a term renaming extends in a natural way to rename also norm definitions. We simply apply term renaming to the terms occurring in the definition.

EXAMPLE 4.7. (Norm renaming) Consider the following semi-linear norm (below on the left), which corresponds to the type-based norm $\|_{d t}$ from Example 4.2. Its j-renamed norm $\Delta^{j}\left(\left.\right|_{d}\right)$, denoted as $\left.\right|_{d_{j}}$, is depicted below (on the right):

$$
H_{d}=\left\{\begin{array}{ll}
1+\left|t^{\prime}\right|_{d} & \text { if } t=d\left(t^{\prime}\right) \\
0 & \text { otherwise }
\end{array} \quad||_{d_{j}}= \begin{cases}1+\left|t^{\prime}\right|_{d_{j}} & \text { if } t=d_{j}\left(t^{\prime}\right) \\
0 & \text { otherwise }\end{cases}\right.
$$

Now we are in a position to define a norm as a combination of some other norms. Basically, we rename the individual norms apart and take their union.

Definition 4.3. (A combined norm) Let $\mathcal{N}=\left\{|\cdot|_{1}, \ldots,|\cdot|_{k}\right\}$, and let the norms $H_{i}$ be defined by the set of equations $E_{i}$. The combined norm $\|_{\mathcal{N}}$ is given by its set of equations $E_{\mathcal{N}}$ as follows:

$$
E_{\mathcal{N}}=\bigcup_{i=1}^{k}\left\{\Delta^{i}(e) \mid e \in E_{i}\right\}
$$

The following example illustrates the combination of two semi-linear norms corresponding to those from Example 4.2.

ExAmple 4.8. Consider the semi-linear norm $\left.\right|_{d}$ from Example 4.7 corresponding to the type-based norm $\psi_{d t}$ of Example 4.2. Consider also the following semilinear norm $\psi_{e}$ which corresponds to the type-based norm $\|_{\text {exp }}$ from Example 4.2

$$
H_{e}= \begin{cases}\left|t^{\prime}\right|_{e}+\left|t^{\prime \prime}\right|_{e} & \text { if } t=t^{\prime}+t^{\prime \prime} \text { or } t=t^{\prime} * t^{\prime \prime} \\ \left|t^{\prime}\right|_{e} & \text { if } t=d\left(t^{\prime}\right) \text { or } t=e\left(t^{\prime}\right) \\ 0 & \text { otherwise }\end{cases}
$$

The combined norm $\left.\right|_{\mathcal{N}}$ of $\psi_{d}$ (from Example 4.7) and $\psi_{e}$ is obtained by joining together the definitions of the renamed norms $\Delta^{1}\left(||_{d}\right)$ and $\Delta^{2}\left(||_{e}\right)$ : 


$$
\left.\right|_{\mathcal{N}}= \begin{cases}1+\left|t^{\prime}\right|_{\mathcal{N}} & \text { if } t=d_{1}\left(t^{\prime}\right) \\ \left|t^{\prime}\right|_{\mathcal{N}}+\left|t^{\prime \prime}\right|_{\mathcal{N}} & \text { if } t=t^{\prime}+{ }_{2} t^{\prime \prime} \text { or } t=t^{\prime} *_{2} t^{\prime \prime} \\ \left|t^{\prime}\right|_{\mathcal{N}} & \text { if } t=d_{2}\left(t^{\prime}\right) \text { or } t=e_{2}\left(t^{\prime}\right) \\ 0 & \text { otherwise }\end{cases}
$$

Note that terms of the form $d(t)$ in the original program are copied to the abstract program either as $d_{1}\left(t^{\prime}\right)$ or $d_{2}\left(t^{\prime \prime}\right)$ (where $t^{\prime}$ and $t^{\prime \prime}$ correspond to renamings of $t$ ), using the above (combined) norm, terms of the form $d_{1}\left(t^{\prime}\right)$ are abstracted using the rule from $\Delta^{1}\left(|\cdot|_{d}\right)$, namely to $1+\left|t^{\prime}\right|_{\mathcal{N}}$, while terms of the form $d_{2}\left(t^{\prime \prime}\right)$ are abstracted using the rule from $\Delta^{2}\left(\left.\right|_{e}\right)$, namely to $\left|t^{\prime \prime}\right|_{\mathcal{N}}$.

Correctness follows because $P$ and $P^{\mathcal{N}}$ have the same termination behaviour and because the analysis for $P$ combining the norms from $\mathcal{N}$ proceeds with exactly the same abstract program as the analysis of $P^{\mathcal{N}}$ with the single norm $\psi_{\mathcal{N}_{\mathcal{N}}}$. Proposition 2.2 can then be applied to establish the correctness of termination analysis.

\section{EXPERIMENTAL EVALUATION}

We have implemented the techniques presented in Sections 3 and 4 and integrated these into the TerminWeb analyser [Taboch et al. 2002]. The modules concerned with user interface and with program abstraction have been extended to handle type information. No other changes were necessary. The implementation effort required only a few hours of coding.

From a theoretical point of view, the analysis based on combination of several norms is always at least as precise as the analyses with each of the individual norms. This claim could be formalised by showing that the single norm analyses are abstractions (in the sense of abstract interpretation) of the combined norm analysis. However this is true only in the absence of the application of the widening operation of the domain of convex polyhedra [Cousot and Halbwachs 1978]. Since this operator is not monotonic and moreover, in our implementation, it depends on the syntactic representation of the linear constraints, we cannot provide any general formal precision claim for this domain. However, in practice, experimentation indicates that combined analyses are no less precise than when using individual norms.

As for efficiency, combined analysis increases the number of arguments in the predicates of the abstract program. This could potentially lead to an exponential increase in the number of abstract binary clauses derived during analysis. Moreover, the constraints between the (increased number of) arguments in each binary clause may become more complex and hence the abstract operations on them more costly. However, in practice, experimentation indicates that the overhead for combined analysis is reasonable and that the number of binary clauses increases slightly (by 1) only in 5 of the benchmark programs.

We have performed an experimental evaluation focusing on cost and precision of our techniques based on a collection of 45 typed logic programs. Table I summarises these experiments and contains three blocks of entries. An extended version of Table I together with the source programs is available from http://www.cs.bgu.ac. 


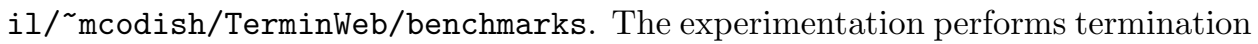
inference [Mesnard 1996] using TerminWeb as described in [Genaim and Codish 2005]. For a given program the analyser infers a class of queries which is guaranteed to terminate. The extended table also indicates for each program the class of queries determined by the analyser to be terminating. The programs in the first block are adapted from [Apt 1997]. Type definitions and declarations were added manually and symbolic arithmetic introduced as TerminWeb does not support native Prolog arithmetic. These are the same programs used in the experimentation reported in Table 1 of [Lagoon et al. 2003]. The programs in the second block include the examples from [Vanhoof and Bruynooghe 2002; Genaim et al. 2002; Martin et al. 1996; Bruynooghe et al. 2002] and correspond to those given in Table 2 of [Lagoon et al. 2003]. The programs in the third block are those from this paper. The experiments were performed using SICStus 3.10.0 running on an Intel Pentium III 933MHz, 640MB RAM, Linux 2.4.22.

The first column of Table I indicates the benchmark program name together with the number of types defined in the program. For this collection, the number of types ranges from 1 to 4 . The columns headed $\mathbf{l l}$ and ts indicate the analysis times (in milliseconds) using the list-length and term-size norms respectively. The column headed ts $\times$ ll specifies the analysis time for the combined list-length and term-size analysis where $\checkmark$ indicates that the combined analysis is more precise than both of the list-length and term-size analyses. Similarly, the columns headed $\mathbf{T}_{\mathbf{1}}, \ldots, \mathbf{T}_{\mathbf{4}}$ indicate the analysis times using type-based norms for the (up to four) types defined in the programs. The column headed $\boldsymbol{\Pi} \boldsymbol{T}_{\mathbf{i}}$ indicates the analysis time using the corresponding combined type-based norms where $\checkmark$ indicates that the combined analysis is more precise than each of the individual type-based analyses $\left(\mathbf{T}_{\mathbf{1}}, \ldots, \mathbf{T}_{\mathbf{4}}\right)$. The symbol $\oplus$ indicates that the combined type-based analysis is more precise than the combined $\mathbf{t s} \times \mathbf{l l}$ analysis.

For all analyses, the analyser is set to allow 2 iterations before applying widening (except for mergesort where 4 iterations are allowed). Note that the only operation performed manually is the addition of the type declarations.

Analysis Time. For 39 of the benchmark programs the run-time of the combined analysis $(\mathbf{t s} \times \mathbf{l l})$ is between 1-2 times larger than the sum of the run-times of the individual analyses and for the other 6 programs it is 2-3 times larger. For 26 of the benchmark programs the run-time of the combined type-based analysis $\left(\boldsymbol{\Pi} \mathbf{T}_{\mathbf{i}}\right)$ is between 1-2 times larger than the sum of the run-times of the individual typebased analyses. For 15 programs it is 2-3 times larger, for 2 programs it is $3-4$ times larger, and for the other two programs it is about 6 and 7 times larger. The slowdown in the combined analyses stems mainly from new information expressed as size dependencies between the components of the different norms. The number of abstract binary clauses in the combined analyses is basically the same as (the maximal number of those) in the corresponding individual analyses. To be precise there is one additional abstract clause in the combined analysis for only five of the programs (der.pl, g.pl, p.pl, t_nonlin.pl and t.pl).

Analysis Precision. Precision is measured in terms of the class of initial queries for which termination can be guaranteed. We say that one analysis is more precise than another if it can prove termination for a larger class of initial queries. For 


\begin{tabular}{|c|c|c|c|c|c|c|c|c|c|c|}
\hline \multirow{2}{*}{\multicolumn{2}{|c|}{ Bench. (\#Types) }} & \multicolumn{4}{|c|}{ semi-linear norms } & \multicolumn{5}{|c|}{ type-based norms } \\
\hline & & \multirow{2}{*}{$\begin{array}{c}11 \\
20\end{array}$} & \multirow{2}{*}{$\begin{array}{c}\mathbf{t s} \\
20\end{array}$} & \multicolumn{2}{|c|}{ ts $\times 11$} & \multirow{2}{*}{$\begin{array}{c}\mathbf{T}_{\mathbf{1}} \\
20\end{array}$} & \multirow{2}{*}{$\begin{array}{c}\mathbf{T}_{\mathbf{2}} \\
20\end{array}$} & \multirow[t]{2}{*}{$\mathbf{T}_{3}$} & \multirow[t]{2}{*}{$\mathbf{T}_{4}$} & \multirow{2}{*}{$\overline{\Pi T_{i}}$} \\
\hline append.pl & $(2)$ & & & 80 & & & & & & \\
\hline delete.pl & (2) & 30 & 100 & 240 & & 120 & 90 & & & $480 \checkmark \oplus$ \\
\hline frontier.pl & (3) & 50 & 60 & 130 & & 60 & 30 & 50 & & $370 \oplus$ \\
\hline in.pl & (2) & 20 & 70 & 130 & & 70 & 30 & & & $180 \checkmark \oplus$ \\
\hline inorder.pl & (3) & 40 & 70 & 150 & & 60 & 30 & 40 & & $370 \oplus$ \\
\hline insert.pl & (2) & 30 & 100 & 190 & & 100 & 50 & & & $310 \checkmark \oplus$ \\
\hline length.pl & (3) & 10 & 20 & 90 & & 10 & 20 & 20 & & $100 \oplus$ \\
\hline length1.pl & (3) & 10 & 20 & 30 & & 20 & 10 & 20 & & 110 \\
\hline less.pl & (1) & 0 & 20 & 50 & & 20 & & & & 30 \\
\hline list.pl & $(2)$ & 0 & 10 & 30 & & 10 & 0 & & & 10 \\
\hline map_color.pl & (4) & 70 & 80 & 220 & $\checkmark$ & 90 & 90 & 40 & 40 & 770 \\
\hline maximum.pl & (1) & 10 & 30 & 70 & & 30 & & & & 30 \\
\hline member.pl & (2) & 10 & 20 & 40 & & 20 & 20 & & & 60 \\
\hline mergesort.pl & (2) & 260 & 310 & 840 & $\checkmark$ & 280 & 280 & & & $860 \checkmark$ \\
\hline minimum.pl & (2) & 10 & 30 & 40 & & 10 & 20 & & & $70 \checkmark \oplus$ \\
\hline mult.pl & (1) & 20 & 50 & 110 & & 60 & & & & 60 \\
\hline naive_reverse.pl & (2) & 40 & 30 & 120 & & 50 & 30 & & & 130 \\
\hline numeral.pl & (1) & 0 & 10 & 10 & & 10 & & & & 10 \\
\hline ordered.pl & (2) & 20 & 40 & 80 & & 10 & 30 & & & 80 \\
\hline palindrome.pl & (2) & 30 & 30 & 80 & & 30 & 20 & & & 100 \\
\hline permutation.pl & (2) & 60 & 40 & 170 & & 60 & 50 & & & 200 \\
\hline permutation1.pl & (2) & 40 & 40 & 120 & & 50 & 40 & & & 200 \\
\hline prefix.pl & (2) & 30 & 20 & 90 & & 30 & 20 & & & 90 \\
\hline quicksort.pl & (2) & 170 & 190 & 520 & & 460 & 180 & & & 2020 \\
\hline reverse.pl & $(2)$ & 30 & 30 & 80 & & 30 & 20 & & & 100 \\
\hline search_tree.pl & (2) & 40 & 250 & 380 & & 200 & 60 & & & 590 \\
\hline select.pl & (2) & 20 & 20 & 60 & & 40 & 30 & & & 150 \\
\hline slowsort.pl & (2) & 80 & 80 & 250 & $\checkmark$ & 70 & 80 & & & $280 \checkmark$ \\
\hline sublist.pl & (2) & 40 & 30 & 90 & & 40 & 20 & & & 110 \\
\hline subset.pl & (2) & 30 & 50 & 90 & & 30 & 40 & & & 120 \\
\hline suffix.pl & (2) & 30 & 20 & 80 & & 40 & 20 & & & 90 \\
\hline sum.pl & (1) & 10 & 30 & 80 & & 30 & & & & 30 \\
\hline tree.pl & (2) & 10 & 10 & 20 & & 10 & 10 & & & 40 \\
\hline tree_member.pl & (2) & 10 & 30 & 60 & & 30 & 30 & & & $110 \oplus$ \\
\hline factor.pl & $\overline{(2)}$ & $\overline{40}$ & $\overline{800}$ & 180 & $\bar{\nabla}$ & $\overline{50}$ & $\overline{880}$ & & & $260 \checkmark$ \\
\hline flat.pl & (3) & 50 & 40 & 140 & $\checkmark$ & 60 & 30 & 50 & & $170 \checkmark \oplus$ \\
\hline flatlength.pl & (4) & 60 & 50 & 210 & $\checkmark$ & 70 & 50 & 40 & 20 & $560 \checkmark \oplus$ \\
\hline g.pl & (3) & 60 & 50 & 170 & & 40 & 50 & 40 & & $390 \oplus$ \\
\hline p.pl & (2) & 20 & 20 & 120 & $\checkmark$ & 20 & 20 & & & $110 \checkmark$ \\
\hline t.pl & (3) & 40 & 60 & 160 & $\checkmark$ & 60 & 40 & 30 & & $330 \checkmark$ \\
\hline ackermann.pl & (1) & 30 & 100 & 310 & & 170 & & & & 180 \\
\hline der.pl & (3) & 10 & 70 & 110 & & 40 & 70 & 40 & & $920 \checkmark \oplus$ \\
\hline p_nonlin.pl & (2) & 30 & 90 & 240 & $\checkmark$ & 50 & 80 & & & $290 \checkmark$ \\
\hline parse.pl & $(2)$ & 50 & 130 & 450 & & 70 & 120 & & & 480 \\
\hline transpose.pl & (3) & 70 & 130 & 320 & $\checkmark$ & 110 & 90 & 40 & & $1710 \checkmark \oplus$ \\
\hline
\end{tabular}

Table I. Performance and precision comparison of termination analysis in different settings (times are in milliseconds): $\mathbf{t s}=$ term-size, $\mathbf{l}=$ list-length, $\mathbf{t s} \times \mathbf{l l}=$ combination of term-size and list-length, $\mathbf{T}_{\mathbf{1}}-\mathbf{T}_{\mathbf{4}}=$ type-based norms, $\mathbf{\Pi} \mathbf{T}_{\mathbf{i}}=$ combination of type-based norms, $\checkmark=$ The combined analysis is more precise than all corresponding single norm analyses, $\oplus=\mathbf{\Pi} \mathbf{T}_{\mathbf{i}}$ is more precise than ts $\times \mathbf{l l}$

all of the experiments, the combined analyses are no less precise than when using individual norms. Moreover, the combined type-based analyses are always at least as precise as the individual and combined list-length and term-size analyses.

The symbols $\checkmark$ in the column ts $\times$ ll indicate that for 10 of the benchmark programs, the combined $(\mathbf{t s} \times \mathbf{l l})$ analysis is more precise than both of the individual list-length and term-size analyses. The symbols $\checkmark$ in the column $\mathbf{\Pi T}_{\mathbf{i}}$ indicate that for 14 of the benchmark programs, the combined type-based analysis is more precise than each of the individual type-based analyses. The symbol $\oplus$ in the column $\boldsymbol{\Pi T}_{\mathbf{i}}$ indicates that for 15 benchmark programs the combined type-based analysis is more 
precise than the combined ts $\times \mathbf{l l}$ analysis.

For 14 out of the 23 benchmarks for which there is a slowdown of a factor greater than two for a combined analysis there is in return a gain in precision. In the other 9 of the benchmarks for which there is such a slowdown, this is due to the additional information derived (dependencies between arguments corresponding to different norms) which does not contribute to precision in terms of proving termination for more queries.

Comparison with [Lagoon et al. 2003]. A recent work [Lagoon et al. 2003] takes an approach similar to our combination of type-based norms. In this work the authors integrate the treatment of polymorphic types.

The set of benchmarks in the first two blocks of Table I correspond to those in Tables 1 and 2 of [Lagoon et al. 2003]. With respect to performance, our results are comparable to those presented in [Lagoon et al. 2003] (except for quicksort since we added symbolic arithmetic). It is difficult to compare raw timings as the experiments are performed on different machines, using different versions of Prolog and based on different libraries for manipulating constraints. However, timings are similar with regards to the overhead for applying (combined) type-based analyses in comparison to individual list-length and term-size analyses.

For precision, we report the same improvements as in [Lagoon et al. 2003] where their combined type-based analysis is compared to individual term-size and listlength analyses. They indicate for each program if precision of type-based analysis is improved with respect to list-length and with respect to term-size analyses. In their Table 1 (corresponding to the first block of programs in our Table I) there are 12 programs for which there is such an improvement, indicated in their table by a $(\checkmark)$. In our case this means that either there is an improved precision for the combined ts $\times \mathbf{l l}$ analysis with respect to the individual list-length and termsize analyses (a $\checkmark$ in the column ts $\times \mathbf{l l}$ ) or there is an improved precision for the combined type-based analysis with respect to the combined $\mathbf{t s} \times \mathbf{l l}$ analysis (an $\oplus$ in the column $\boldsymbol{\Pi}_{\mathbf{i}}$ ). In the first block of programs in our Table I there are 12 programs indicated to have this kind of precision improvement. There are slight differences between the results in the two papers: The program named query in [Lagoon et al. 2003] corresponds to running map_color/2 for a specific input which is already included in our version of map_color.pl (and hence this example is excluded from our table); Other differences (in the programs search_tree.pl, mergesort.pl and slowsort.pl) stem from our use of symbolic arithmetic. The programs in Table 2 of [Lagoon et al. 2003] correspond to those in the second block in our Table I. For these programs we report the same precision improvements.

We note that the analyser described in [Lagoon et al. 2003] cannot prove termination for three of the programs in the third block of our Table I. These are the programs from Examples 2.3, 4.1 and 4.2. For these examples an analyser based on global termination conditions should support constraint solving with lexicographic orders. Our analyser succeeds to prove termination for these examples, without the support of lexicographic orders, due to the use of local termination conditions. 


\section{RELATED WORK}

The use of types to refine other program analyses has recently been considered in [Bruynooghe et al. 2001] and [Lagoon and Stuckey 2001]. The basic idea is to refine the way the abstract unification operation for a given domain is defined so as to consider that if a program is well-typed then only sub-terms of the same type can be unified. Our idea of multiplying the arguments of predicates - one copy for each type constituent - resembles the approach used in [Lagoon and Stuckey 2001] where for each type the corresponding copy of an argument contains its sub-terms of that type. These works improve on the observations made in [Decorte et al. 1997] where the authors abstract each predicate argument with respect to a single but possibly different type.

The idea of using type information to define norms has previously been studied by Bossi et al. [Bossi et al. 1992], Martin et al. [Martin et al. 1996], and by Decorte et al. [Decorte et al. 1993; 1996; 1997]. Our approach builds primarily on the technique of Decorte et al.

Decorte et al. observe in [Decorte et al. 1993] and in [Decorte et al. 1996] that different predicate arguments can be measured by different (type-based) norms. The authors also observe that inter-argument size relations between different norms can provide useful information. Their work suffers from two restrictions. First, at most one type-based norm can be applied to a term of a given type. Second, as reported in [Decorte et al. 1997], the use of different norms for different arguments, in their approach, renders the computation of inter-argument size relations far from trivial. They propose a partial solution based on a notion of re-execution. In contrast we propose to combine different norms by duplicating predicate arguments and to apply the analyses simultaneously. A similar idea was suggested in [King et al. 1997] in the context of lower-bound time-complexity analysis to increase the precision of inter-argument size relations.

So far experiments with the approach presented in this paper have focused on the analysis of well-typed programs where types are declared by the user. Type inference systems such as those described in [Gallagher and Puebla 2002; Vaucheret and Bueno 2002] are capable of deriving a monomorphic type for a given program. While inferred types for a program are guaranteed to be correct (that is, the types over-approximate the success set of the program) they do not necessarily give a well-typing. One solution is to transform the inferred types into a well-typing. However, there is a simple rudimentary way of ensuring that type-based termination analysis is still correct for ill-typed programs. One isolates the ill-typing to ensure that it always occurs in unifications of the form $X: \tau_{1}=Y: \tau_{2}$. This is always possible. Indeed if an atom of another form has an argument $X$ that is ill-typed, it can be replaced by a new variable $Y$ that has the correct typing and an extra (ill-typed) unification $X: \tau_{1}=Y: \tau_{2}$ can be added without affecting the termination behaviour of the program. Then, after removal of all ill-typed unifications, the type-based termination analysis can be applied. If termination can be proved, then the original program terminates. However, it could be that the removed unifications contain size-relations that are crucial for proving the termination. A refined approach is described in a recent work [Lagoon et al. 2003]. If the unification $X: \tau_{1}=Y: \tau_{2}$ succeeds, then the resulting value is correctly typed by 
both $\tau_{1}$ and $\tau_{2}$. Comparing both type definitions, one can build size relationships between their constituents. These relationships preserve information that is lost when discarding ill-typed unifications. The authors of [Lagoon et al. 2003] were also the first to report an extensive evaluation of type-based termination analysis of programs with declared types. They also report a few experiments where types were inferred using the approach of [Gallagher and de Waal 1994]. In several cases, the termination conditions are weaker than those obtained with declared types. This suggests that the success set approximations inferred by [Gallagher and de Waal 1994] are not the right types for use in type-based termination analysis. Very recently a method has been developed to infer a well-typing from a given program [Bruynooghe et al. 2005]. Preliminary results on the programs listed in Table I indicate that termination conditions derived from the inferred type are as strong as those obtained from the declared types.

An initial study about the extension of type-based termination analysis to polymorphic types is reported in [Bruynooghe et al. 2002]. It overlooks a problem of interference between different type constituents. A solution is offered in [Bruynooghe et al. 2003]. A slightly different approach is taken by the authors of [Lagoon et al. 2003]; while more convenient -it does not require knowledge of an upper bound on the number of constituents in the type of a call- it can cause the polymorphic analysis to be less precise than the corresponding meromorphic analysis. An advantage of the latter work is that it can handle unifications $X=Y$ where $X$ and $Y$ have a different type, a feature also exploited in handling calls with a type that is an instance of the declared polymorphic type.

The idea of applying local termination conditions originates in the TermiLog system [Lindenstrauss and Sagiv 1997]. It is used in the TerminWeb analyser and is also considered in the functional setting as described in [Lee et al. 2001]. In contrast, the work described in [Lagoon et al. 2003] is based on an analyser which checks global termination conditions. There is a practical advantage in basing implementations on the local approach. This is because the termination conditions take a simpler form and are easier to prove. We have seen this in the context of Example 2.3 (and others), where the global approach requires lexicographic orderings to be considered. It is also the case in Example 4.1. Here, with the local approach, termination follows (for the combined term-size and list-length analysis) using the abstract domain of monotonicity constraints. The global approach requires the identification of a termination condition of the form $f(p(x, y))=x+2 y$ which can be done using a richer abstract domain.

The use of different level mappings for different loops also increases substantially the modularity of the proofs. In previous works addressing modularity, although one clause at a time was considered, the same level mappings were used across the whole program or, in a recent work [Bossi et al. 2002], across all the clauses in one module out of a hierarchy of modules.

\section{CONCLUSION}

This paper describes a technique which enables a termination analyser to consider a combination of several (type-based) norms. The contribution is a powerful technique which stems from two simple ideas: (1) interesting type-based norms can be defined by counting the number of sub-terms of a given type occurring in (typed) 
terms; and (2) replicating the arguments of a predicate (once for each type-based norm) solves many of the problems encountered in previous related work. The advantages follow from three aspects. Firstly, the analysis benefits from taking into account dependencies amongst different measures of the terms, which are contained in the types. Secondly, the analyser considers all measures simultaneously, and so can discover which combination of the measures derived from the program's types is suitable for proving termination. Finally, the implementation becomes straightforward.

The implementation of an analyser which supports the combination of norms and type-based norms is derived from the termination analyser described in [Codish and Taboch 1999] simply by changing the abstraction module and the user interface. No other changes are necessary. When the user provides a program to the analyser, first it infers type-based norms from the program (if the program is typed) and then provides the user with a list of the inferred norms together with a predefined norms collection (like list-length, term-size, etc,), then the user can apply the combined analysis with respect to all type-based norms (the default) or with respect to a subset of the available norms. The analyser can be accessed or downloaded from http://www.cs.bgu.ac.il/ mcodish/TerminWeb. The analyses for the benchmark programs from Table I can be performed by accessing

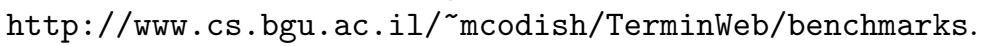

\section{Acknowledgements}

We acknowledge the many fruitful discusions with Amir Ben-Amram, Vitaly Lagoon, Fred Mesnard and Peter Stuckey. Comments from anonymous referees helped to improve and clarify the presentation.

\section{REFERENCES}

Apt, K. R. 1990. Introduction to logic programming. In Handbook of Theoretical Computer Science, J. van Leeuwen, Ed. Vol. B: Formal Models and Semantics. Elsevier, Amsterdam and The MIT Press, Cambridge, 495-574.

Apt, K. R. 1997. From Logic Programming to Prolog. Prentice Hall.

Apt, K. R. And Pedreschi, D. 1993. Reasoning about termination of pure Prolog programs. Information and Computation 106, 1 (Sept.), 109-157.

Benoy, F. AND KInG, A. 1996. Inferring argument size relationships with CLP(R). In Sixth International Workshop on Logic Program Synthesis and Transformation (LOPSTR'96). 204223.

Bossi, A., Cocco, N., Etalle, S., And Rossi, S. 2002. On modular termination proofs of general logic programs. Theory and Practice of Logic Programs 2, 3, 263-291.

Bossi, A., Cocco, N., And FABRis, M. 1991. Proving termination of logic programs by exploiting term properties. In Proceedings of Tapsoft 1991, S. Abramsky and T. Maibaum, Eds. Lecture Notes in Computer Science, vol. 494. Springer-Verlag, Berlin, 153-180.

Bossi, A., Cocco, N., and Fabris, M. 1992. Typed norms. In Proceeedings ESOP '92, B. KriegBrückner, Ed. Lecture Notes in Computer Science, vol. 582. Springer-Verlag, Berlin, 73-92.

Brodsky, A. And Sagiv, Y. 1989. Inference of monotonicity constraints in Datalog programs. In Proceedings of the Eighth ACM SIGACT-SIGART-SIGMOD Symposium on Principles of Database Systems. 190-199.

Bruynooghe, M., Codish, M., Genaim, S., and Vanhoof, W. 2002. Reuse of results in termination analysis of typed logic programs. In Proceedings of The 9th International Static Analysis Symposium, M. Hermenegildo and G. Puebla, Eds. Lecture Notes in Computer Science, vol. 2477. Springer-Verlag, 477-492. 
Bruynooghe, M., Codish, M., Genaim, S., and Vanhoof, W. 2003. A note on the reuse of the results of a termination analysis based on polymorphic types. Tech. rep., Katholieke Universiteit Leuven, Dept. of Computer Science. Report CW 383, http://www.cs.kuleuven.ac.be/publicaties/rapporten/cw/CW383.abs.html.

Bruynooghe, M., Gallagher, J. P., and Van Humbeeck, W. $2005 . \quad$ Inference of well-typings for logic programs with application to termination analysis. Tech. rep., Katholieke Universiteit Leuven, Dept. of Computer Science. Report CW 409 (to appear, Proceedings of the 12th International Static Analysis Symposium, SAS 2005), http://www.cs.kuleuven.ac.be/publicaties/rapporten/cw/CW409.abs.html.

Bruynooghe, M., Vanhoof, W., And Codish, M. 2001. Pos(T): Analyzing dependencies in typed logic programs. In Perspectives of System Informatics, 4th International Andrei Ershov Memorial Conference, (PSI). Lecture Notes in Computer Science, vol. 2244. Springer-Verlag, 406-420.

Codish, M. And Genaim, S. 2003. Proving termination one loop at time. In Proceedings of the 13th International Workshop on Logic Programming Environments, (http://www.cs.kuleuven.ac.be/publicaties/rapporten/cw/CW371.abs.html), F. Mesnard and A. Serebrenik, Eds. 53-64.

Codish, M., Genaim, S., Bruynooghe, M., Gallagher, J., and Vanhoof, W. 2003. One loop at a time. In 6th International Workshop on Termination (http://www.dsic.upv.es/ rdp03/wst/proceedings.html).

Codish, M. And TABOCH, C. 1999. A semantic basis for the termination analysis of logic programs. The Journal of Logic Programming 41, 1, 103-123.

Cousot, P. And HalBwachs, N. 1978. Automatic discovery of linear restraints among variables of a program. In Proceedings of the Fifth Annual ACM Symposium on Principles of Programming Languages. 84-96.

De Schreye, D. And Verschaetse, K. 1995. Deriving linear size relations for logic programs by abstract interpretation. New Generation Computing 13, 02, 117-154.

De Schreye, D., Verschaetse, K., and Bruynooghe, M. 1992. A framework for analyzing the termination of definite logic programs with respect to call patterns. In Proceedings of the International Conference on Fifth Generation Computer Systems. ICOT, Japan, 481-488.

Decorte, S., De Schreye, D., And Fabris, M. 1993. Automatic inference of norms: A missing link in automatic termination analysis. In Logic Programming - Proceedings of the 1993 International Symposium, D. Miller, Ed. MIT Press, Vancouver, Canada, 420-436.

Decorte, S., De Schreye, D., And Fabris, M. 1996. Integrating types in termination analysis. Tech. Rep. CW 222, K.U.Leuven, Department of Computer Science.

Decorte, S., De Schreye, D., and Fabris, M. 1997. Exploiting the power of typed norms in automatic inference of interargument relations. Tech. Rep. CW 246, K.U.Leuven, Department of Computer Science.

Dershowitz, N. 1995. 33 examples of termination. In French Spring School of Theoretical Computer Science Advanced Course on Term Rewriting (Font Romeux, France, May 1993), H. Comon and J.-P. Jouannaud, Eds. Lecture Notes in Computer Science, vol. 909. SpringerVerlag, Berlin, 16-26.

Dershowitz, N., Lindenstrauss, N., Sagiv, Y., and Serebrenik, A. 2001. A general framework for automatic termination analysis of logic programs. Applicable Algebra in Engineering, Communication and Computing 12, 1-2, 117-156.

Dershowitz, N. And Manna, Z. 1979. Proving termination with multiset orderings. Communications of the ACM 22, 465-476.

Gabbrielli, M. and Giacobazzi, R. 1994. Goal independency and call patterns in the analysis of logic programs. In Proceedings of the Ninth ACM Symposium on Applied Computing. ACM Press, 394-399.

Gallagher, J. and Puebla, G. 2002. Abstract Interpretation over Non-Deterministic Finite Tree Automata for Set-Based Analysis of Logic Programs. In Practical Aspects of Declarative Languages, 4th International Symposium, PADL 2002, Portland, OR, USA, January 19-20, 
2002, S. Krishnamurthi and C. R. Ramakrishnan, Eds. Lecture Notes in Computer Science, vol. 2257. Springer, 243-261.

Gallagher, J. P. AND DE WAal, D. A. 1994. Fast and precise regular approximations of logic programs. In Proceedings of the Eleventh International Conference on Logic Programming, P. Van Hentenryck, Ed. The MIT Press, Santa Margherita Ligure, Italy, 599-613.

Genaim, S. And Codish, M. 2005. Inferring termination conditions for logic programs using backwards analysis. TPLP 5, 1-2, 75-91.

Genaim, S., Codish, M., Gallagher, J., and Lagoon, V. 2002. Combining norms to prove termination. In Third International Workshop on Verification, Model Checking and Abstract Interpretation, A. Cortesi, Ed. Lecture Notes in Computer Science, vol. 2294. Springer-Verlag, $126-138$.

Graham, R. L., Rothschild, B. L., And Spencer, J. H. 1980. Ramsey theory. Wiley-Interscience Series in Discrete Mathematics. John Wiley \& Sons, Inc., New York. A Wiley-Interscience Publication.

Hill, P. M. And Topor, R. W. 1992. A semantics for typed logic programs. In Types in Logic Programming, F. Pfenning, Ed. The MIT Press.

KARR, M. 1976. Affine relationships among variables of a program. Acta Informatica 6, 133-151.

King, A., Shen, K., And Benoy, F. 1997. Lower-bound time-complexity analysis of logic programs. In International Symposium on Logic Programming, J. Maluszynski, Ed. MIT Press, $261-276$.

Lagoon, V. 2004. Analysis of typed logic programs. Ph.D. thesis, The University of Melbourne, Australia.

Lagoon, V., Mesnard, F., And Stuckey, P. 2003. Termination analysis with types is more accurate. In Proceedings of the Nineteenth International Conference on Logic Programming, C. Palamidessi, Ed. Lecture Notes in Computer Science, vol. 2916. Springer, 254-268.

Lagoon, V. And Stuckey, P. 2001. A framework for analysis of typed logic programs. In FLOPS. Lecture Notes in Computer Science, vol. 2024. Springer-Verlag, Berlin, 296-310.

LEE, C. S. 2002. Program termination analysis and termination of offline partial evaluation. Ph.D. thesis, University of Western Australia.

Lee, C. S., Jones, N. D., And Ben-Amram, A. M. 2001. The size-change principle for program termination. ACM SIGPLAN Notices 36, 3, 81-92. Proceedings of POPL'01.

Lindenstrauss, N. And Sagiv, Y. 1997. Automatic termination analysis of logic programs. In Proceedings of the Fourteenth International Conference on Logic Programming, L. Naish, Ed. The MIT Press, Leuven, Belgium, 63-77.

Lloyd, J. 1987. Foundations of Logic Programming, $2^{\text {nd }}$ ed. Springer-Verlag, Berlin.

Marriott, K. and Søndergaard, H. 1993. Precise and efficient groundness analysis for logic programs. ACM Letters on Programming Languages and Systems 2, 1-4, 181-196.

Martin, J., King, A., And Soper, P. 1996. Typed norms for typed logic programs. In Logic Programming Synthesis and Transformation, 6th International Workshop, LOPSTR'96, J. P. Gallagher, Ed. Lecture Notes in Computer Science, vol. 1207. Springer-Verlag, 224-238. Available at http://www.cs.ukc.ac.uk/pubs/1996/511.

Mesnard, F. 1996. Inferring left-terminating classes of queries for constraint logic programs. In Proceedings of the 1996 Joint International Conference and Syposium on Logic Programming, M. J. Maher, Ed. The MIT Press, Bonn, Germany, 7-21.

Mesnard, F. and Neumerkel, U. 2001. Applying static analysis techniques for inferring termination conditions of logic programs. In Static Analysis, 8th International Symposium, SAS 2001, P. Cousot, Ed. Lecture Notes in Computer Science, vol. 2126. 93-110.

Mycroft, A. And O'Keefe., R. 1984. A polymorphic type system for Prolog. Artificial Intelligence 23, 3, 295-307.

Ramsey, F. 1930. On a problem of formal logic. In Proceedings London Mathematical Society. Vol. 30. 264-286.

Serebrenik, A. And De Schreye, D. 2001. Non-transformational termination analysis of logic programs, based on general term-orderings. In Logic Based Program Synthesis and Transfor- 
mation, 10th International Workshop, LOPSTR 2000 London, UK, July 24-28, 2000, Selected Papers, K.-K. Lau, Ed. Lecture Notes in Computer Science, vol. 2042. Springer, 69-85.

Somogyi, Z., Henderson, F., And Conway, T. 1996. The execution algorithm of Mercury, an efficient purely declarative logic programming language. Journal of Logic Programming 29, 1-3, $17-64$.

Sterling, L. And Shapiro, E. 1994. The Art of Prolog, second ed. Mit Press.

Taboch, C., Genaim, S., And Codish, M. 2002. Terminweb: Semantic based termination analyser for logic programs. http://www.cs.bgu.ac.il/ moodish/TerminWeb.

Vanhoof, W. And Bruynooghe, M. 2002. When size does matter. In Logic Based Program Synthesis and Transformation, 11th International Workshop, LOPSTR 2001, A. Pettorossi, Ed. Lecture Notes in Computer Science, vol. 2372. 129-147.

VAucheret, C. And Bueno, F. 2002. More precise yet efficient type inference for logic programs. In Proceedings of The 9th International Static Analysis Symposium, M. Hermenegildo and G. Puebla, Eds. Lecture Notes in Computer Science, vol. 2477. Springer-Verlag, 102-116. 